\title{
Time-to-Contact Estimation of Accelerated Stimuli Is Based on First-Order Information
}

\author{
Nicolas Benguigui \\ University of Paris-Sud (XI)
}

\author{
Hubert Ripoll \\ University of the Mediterranean
}

\author{
Michael P. Broderick \\ Arizona State University
}

\begin{abstract}
The goal of this study was to test whether 1st-order information, which does not account for acceleration, is used (a) to estimate the time to contact (TTC) of an accelerated stimulus after the occlusion of a final part of its trajectory and (b) to indirectly intercept an accelerated stimulus with a thrown projectile. Both tasks require the production of an action on the basis of predictive information acquired before the arrival of the stimulus at the target and allow the experimenter to make quantitative predictions about the participants' use (or nonuse) of 1st-order information. The results show that participants do not use information about acceleration and that they commit errors that rely quantitatively on 1st-order information even when acceleration is psychophysically detectable. In the indirect interceptive task, action is planned about $200 \mathrm{~ms}$ before the initiation of the movement, at which time the 1st-order TTC attains a critical value.
\end{abstract}

In interceptive actions, the essential problem to be resolved consists of coordinating the action with the approach of the object. In the temporal domain, it is generally accepted that action timing requires access to information that accounts for the time to contact (TTC), that is, the time remaining before the object reaches the actor or a point in space where it is to be intercepted (e.g., Tresilian, 1990). This issue has been addressed in a large number of studies, beginning with the seminal work by Lee $(1976,1980)$. Lee (1980) proposed that the timing of interceptive actions could be based on the optic variable $t a u, \tau(\varphi)$, that is, the inverse of the rate of expansion of the angle $\varphi$ formed by the object with respect to the observer. This variable and the conditions under which this variable is used have been pursued over the course of the past 25 years (e.g., Bootsma \& Oudejans, 1993; Lee \& Young, 1985; Savelsbergh, Whiting, \& Bootsma, 1991), but some recent studies have raised questions about the hypothesis (Michaels, Zeinstra, \& Oudejans, 2001; Smeets, Brenner, Trébuchet, \& Mestre, 1996; Tresilian, 1995, 1999a; Wann, 1996). The debate is open and, as

Nicolas Benguigui, Center for Research in Sport Sciences, University of Paris-Sud (XI), Orsay, France; Hubert Ripoll, Sport and Adaptation Laboratory, University of the Mediterranean, Marseille, France; Michael P. Broderick, Motor Control Laboratory, Arizona State University.

We thank Benoît Bardy, Simone Caljouw, Gilles Montagne, and Bill Warren for helpful comments on earlier versions of this article. We are also very grateful for the time taken by the three referees, Heiko Hecht, James Tresilian, and Claire Michaels; their detailed comments helped in shaping the arguments in this article.

Correspondence concerning this article should be addressed to Nicolas Benguigui, Université Paris-Sud, Division STAPS, Centre de Recherche en Sciences du Sport, Bâtiment 335, 91405 Orsay Cedex, France. E-mail: nicolas.benguigui@staps.u-psud.fr
Tresilian (1995, 1999a) has pointed out, the experimental findings obtained in this area are quite various and the theoretical picture is still far from complete.

One of the issues that remains to be explored concerns how humans adapt their actions to objects having accelerating trajectories (e.g., Dubrowski \& Carnahan, 2002; Tresilian, 1999a). ${ }^{1}$ One can easily observe that, in a nonartificial environment, the velocity of moving stimuli is rarely constant. More often, the trajectories encountered are either accelerating because of muscular action or gravitation or decelerating because of air resistance, friction, or gravitation. It seems reasonable, as Rosenbaum (1975) proposed, that the individual adapted to his or her environment uses strategies that take account of the various acceleration characteristics of moving objects.

With accelerated objects, one can also test the validity of Lee's (1976) hypotheses because $\tau(\varphi)$ is a first-order information that does not account for acceleration. Theoretically, two hypotheses can be proposed to explain the timing of interceptive actions with accelerated objects (e.g., Bootsma, Fayt, Zaal, \& Laurent, 1997; Michaels et al., 2001): (a) The actor uses a first-order TTC information (TTC1), for example, $\tau(\varphi)$, and continuously regulates his or her action on the basis of this variable or (b) the actor is capable of using information about the acceleration itself and uses a second-order TTC information (TTC2) that permits "perfect" timing.

\footnotetext{
${ }^{1}$ In this article, the term acceleration is used, depending on the context, either (a) as a generic term for constant variation in speed across time (i.e. both increases and decreases in speed) or (b) to denote an increase in speed. To express specifically a decrease in speed, we use the term deceleration.
} 


\section{Timing of Interceptive Actions With Accelerated Trajectories: TTC1 Hypothesis}

When an object moves at a constant velocity, TTC1 is equal to TTC. However, when an object is moving with accelerating trajectories, TTC1 does not define the observer-object temporal relationship as TTC1 does not account for acceleration. This is what led Tresilian $(1994 a, 1995)$ to define this variable as a first-order approximation of TTC. The issue is whether the information specifying this approximation would be useful or sufficient to intercept or adapt movement to an accelerating object. It has been hypothesized that such information could be sufficient if there were a continuous coupling between the perceptual and the motor systems permitting online adjustment of movement characteristics as a function of TTC1 (e.g., Bootsma et al., 1997; Lee, Young, Reddish, Lough, \& Clayton, 1983; Tresilian, 1999a). One fact that gives impetus to this hypothesis is that TTC1 converges to TTC as the object approaches the point of contact (see Figure 1).

From this perspective, Lee et al. (1983) evaluated the TTC1 hypothesis by analyzing the timing that people use in striking an accelerating ball. The task consisted of leaping to punch a falling ball that was released from three different heights (3.0, 5.0, $7.2 \mathrm{~m})$. Two main results were interpreted as evidence that people use continuous visual guidance of the action relative to first-order information- $\tau(\varphi)$, according to Lee et al. First, they showed that each of the different stages of the action was longer when the falling time was longer (i.e., when the drop height was higher). If the action had been geared to TTC, there should have been no differences between the drop heights. Second, Lee et al. plotted the knee and elbow angles against TTC1 and TTC. They showed that the angle-time functions of the three different height conditions lie much closer to the TTC1-time function than to the TTC-time function. ${ }^{2}$

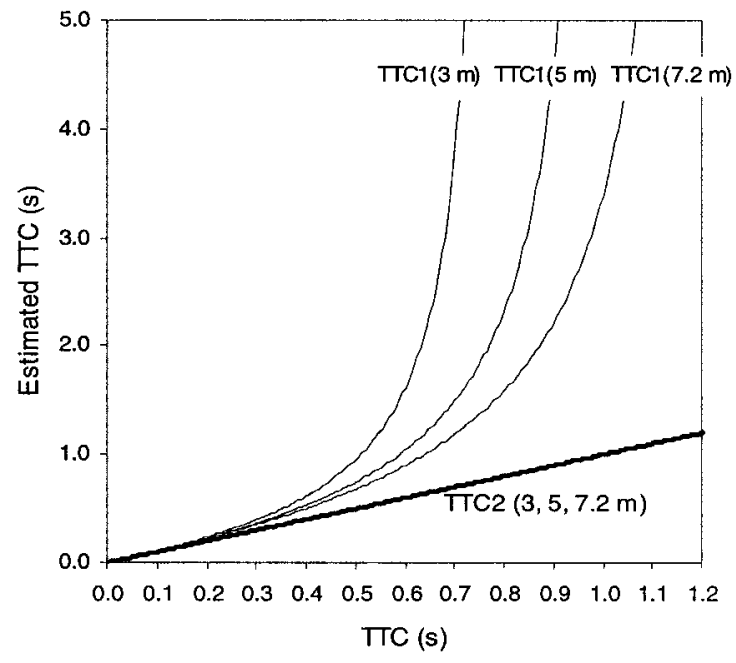

Figure 1. The evolution of TTC1 and TTC2 as a function of TTC, according to data from Lee et al. (1983). In this example, a ball falls vertically because of the force of gravity $\left(g=9.81 \mathrm{~m} / \mathrm{s}^{2}\right)$ for three different drop heights $(3,5$, and $7.2 \mathrm{~m})$. Note in this example that TTC2 is equal to TTC because the accelerations are constant. As TTC diminishes, the difference between TTC 1 and TTC 2 diminishes. TTC $=$ time to contact.
Generalizing the TTC1 hypothesis to nonradial approaches, Bootsma and colleagues (Bootsma et al., 1997; Bootsma \& Oudejans, 1993) proposed that TTC is specified by information combining the relative velocity of the expansion of the optical contour of a moving object $(\dot{\varphi})$ with the relative velocity of the contraction of the visual angle between the object and the point of interception $(\dot{\theta})$. This information variable, $\tau(\varphi, \theta)$, specifying $\operatorname{TTC} 1(x)$ at any point in space, is formalized by $1 / \tau(\varphi, \theta)=\dot{\varphi} / \sin \varphi-\dot{\theta} / \sin \theta=$ $-1 / \operatorname{TTC} 1(x)$, where $\dot{\varphi}$ is the inverse of $\tau(\varphi)$ as defined by Lee (1976) when $\varphi$ is small (when $\varphi \leq 10^{\circ}, \sin \varphi \approx \varphi$ ), $\theta$ corresponds to the angle formed at the participant's observation point by the object and the point of interception, and $\dot{\theta}$ corresponds to the variation of this angle as a function of time (see Figure 2).

On the basis of this relationship, Bootsma and Oudejans (1993) showed, in a forced-choice task in which the participants had to predict as quickly as possible the order of arrival of two stimuli moving toward the same target, that neither the acceleration nor the deceleration were accounted for. The participants committed more errors of judgment when one of the two stimuli or both stimuli were accelerating.

These results were partially confirmed by Kaiser and Hecht (1995, Experiment 1) in a prediction-motion (PM) task. In this experiment, which used a computer simulation, the participants were required to estimate the arrival time of an approaching target "star" that was occluded before arrival. The approach of the "star" was either accelerating, decelerating, or at constant velocity. These different conditions allowed experimental prediction of errors: overestimates of TTC in the case of acceleration and underestimates in the case of deceleration. The predictions were based on the assumption that people do not account for acceleration but rather estimate TTC on the basis of first-order information. The results of the experiment confirmed the predictions with respect to the deceleration condition but not the acceleration condition. To explain this absence of overestimation in the latter condition, Kaiser and Hecht suggested that the values of acceleration and duration of occlusion were not sufficient to give rise to the results expected in such a situation. An additional explanation could be linked to the variability inherent to this kind of task and specifically when there is direct approach (see Schiff \& Oldak, 1990, for a discussion).

Even though several studies have suggested that interceptive actions do not take account of acceleration and that the timing of these actions is based on TTC1, certain results have nevertheless raised questions about these conclusions. These questions can be considered both empirically and theoretically. The empirical issue was initially raised by studies that suggest that acceleration is used for timing interceptive actions (Rosenbaum, 1975; McIntyre, Zago, Berthoz, \& Lacquaniti, 2001; Lacquaniti, Carozzo, \& Borghese, 1993; Lacquaniti \& Maioli, 1989a, 1989b). The theo-

\footnotetext{
${ }^{2}$ As Michaels et al. (2001) remarked, this study had a significant impact on the perception-action community. It was followed by several studies, some of which are described in this article, that confirmed and enlarged on the TTC1 hypothesis. We note also that the Lee et al. (1983) study was criticized on methodological and theoretical grounds (e.g., Tresilian, 1993 , Wann, 1996) and that more recently, Michaels et al. performed a study using the same experimental paradigm that raised questions about the Lee et al. conclusions. This last study is described in more detail below in the next section.
} 


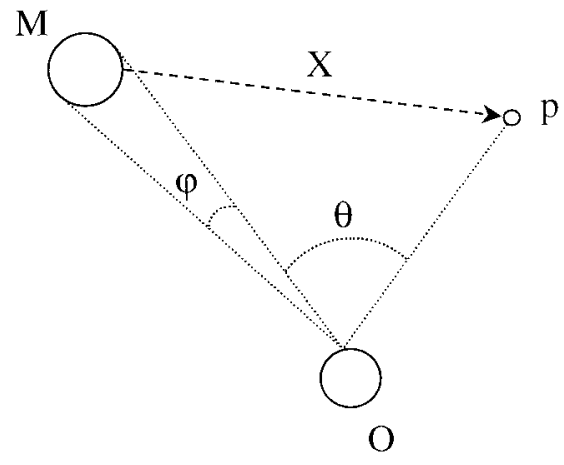

Figure 2. Geometrical relations between an observer $(\mathrm{O})$ and a moving object (M) moving a distance $(\mathrm{X})$ toward a point $(\mathrm{p})$.

retical issue is raised by authors who have questioned the general and exclusive character of the tau hypothesis and suggested that other sources of information could be used to time actions (e.g., Michaels et al., 2001; Tresilian, 1995, 1999a, 1999b).

\section{Timing of Interceptive Actions With Accelerated Trajectories: TTC2 Hypothesis}

Theoretically, it is possible to formalize second-order information that humans could use in order to judge TTC of an accelerating object (Lee et al., 1983). For example, the visual system might take the second derivative of perceived position with respect to time, or it might take the derivative of perceived velocity. It is also possible to define an optic variable directly specifying an object's acceleration. Bootsma and Peper (1992) formalized an optic quantity to specify TTC2 based on the derivative of tau with respect to time, that is, $\dot{\tau}$ : TTC2 $=\tau \cdot[1+\sqrt{1-2 \cdot(\dot{\tau}+1)}] \div$ $(\dot{\tau}+1)$.

Having derived this mathematical possibility (see also Tresilian, 1994a, Appendix A, for a similar formalization), Bootsma and Peper (1992) reflected on the benefit actually accruing to an actor using this variable. They argued that use of TTC 2 would necessitate, as would use of TTC1, continuous regulation of action, insofar as it appears that in nonartificial situations, uniform accelerations are as rare as movements at constant velocity. To address these theoretical questions, we turn to studies that have examined whether people do base their actions on information of acceleration.

Rosenbaum (1975) used a PM task to determine whether humans are capable of using information about acceleration to extrapolate object movement. The task consisted of estimating, by pushing on a button, the moment of a ball's arrival at a target after occlusion of the last part of the ball's trajectory. In this experiment (i.e., Experiment 2), constant velocities $(0.19,0.43$ and $0.73 \mathrm{~m} / \mathrm{s}$ ) and accelerations $\left(0.23,0.39\right.$ and $\left.0.44 \mathrm{~m} / \mathrm{s}^{2}\right)$ were tested. The results showed that the participants were as accurate in their estimations of accelerating objects as of those with constant velocity, suggesting access to information about acceleration. Nevertheless, it is possible that the absence of difference between the velocity and acceleration conditions was due to the low accelerations used (Kaiser \& Hecht, 1995).

Lacquaniti and Maioli (1989a, 1989b) conducted a series of studies in which EMG timing of catching free-falling balls was analyzed. The results suggested that in situations of free fall in which acceleration is produced by the force of earth's gravity $(g)$, it could be possible for humans to learn how to use this value in the timing of interceptive actions (see also Lacquaniti et al., 1993; Tresilian, 1993, 1997, 1999b). Velocity ( $v$ ) information, which varies as a function of time $(t)$, could then be used to time movements on the basis of a temporal variable of the form TTC2 $=v(t) / g$ (Lacquaniti et al., 1993). Using the same logic, Tresilian (1999b) suggested that TTC2 could also be determined with respect to the force of gravity $(g)$ and to release height $(h)$ under the form of the equation TTC $2=\sqrt{2 \cdot h / g}$.

The possible use of such strategy was tested in a recent study by McIntyre et al. (2001) in which astronauts had to catch falling balls in a $1-g$ condition (on earth) and a $0-g$ condition (during the flight). In this experiment, the ball was projected downward with three initial speeds $(0.7,1.7$, and $2.7 \mathrm{~m} / \mathrm{s})$ from a starting point $1.6 \mathrm{~m}$ above the hand. In the $0-g$ condition, the anticipatory responses occurred earlier than in the $1-g$ condition (i.e., at a greater TTC than in the 1- $g$ condition). These results led McIntyre et al. to reject both (a) control of the anticipatory responses on the basis of TTC1 information (in this case, the anticipatory responses should have occurred in the $0-g$ condition later than they did) and (b) direct perception of the acceleration of the ball (in this case, no difference should have been observed between the $0-g$ and $1-g$ conditions). McIntyre et al. concluded that these results were evidence of the use of a second-order internal model of gravity in interceptive timing.

It may well be that McIntyre et al. (2001) were correct in their interpretation of their elegant experiment. On the other hand, their results also suggest that the nature of the information used is task specific and that it is difficult to draw general conclusions. A priori knowledge of the trajectory of a moving object could be used in the timing of interceptive action and different task constraints could give rise to different sources of information (Cutting, 1986; Tresilian, 1994a, 1995, 1999a). Both issues are certainly relevant to evaluating Lee's $(1976,1980)$ concept of tau.

From this perspective, Michaels et al. (2001) replicated the ball-punching experiment of Lee et al. (1983). The first step taken in their study was to eliminate the experimental problems identified in the earlier study (see also Tresilian, 1993, and Wann, 1996, for discussion about the study's methodological problems).

Overall, the results showed that, although part of the analysis showed results compatible with the use of $\tau(\varphi)$, the sources of information susceptible to utilization were more numerous than identified by Lee et al. (1983). First, a participant-by-participant analysis shows that the optic variable $\dot{r}$ (i.e., the velocity of the dilation of the ball) could explain the initiation of movement. This analysis showed that for each participant there appears to be a point of convergence of $\dot{r}$ corresponding to a critical value of this variable related to the initiation of flexion movement. Movement began 150-275 ms (the participants differed) after $\dot{r}$ had attained a critical value. Second, there were inter-individual differences in the use of optic variables. For one of the participants, the variable $\dot{r}$ seems to be able to explain the kinematics of the variation of elbow angle. For the other four, there seems to be a relation between the variation of both $\tau(\varphi)$ and $\tau(\varphi)$, and the variation of elbow angle. The crucial point of Michaels et al. (2001) concerns the many sources of information that could be used in interceptive actions. 


\section{Perceiving the Acceleration of a Moving Stimulus}

A related question is whether the possible identification of acceleration influences the timing of interceptive actions. Sensitivity to acceleration has been tested in a number of psychophysics experiments (e.g., Babler \& Dannemiller, 1993; Brouwer, Brenner, \& Smeets, 2002; Calderone \& Kaiser, 1989; Regan, Kaufman, \& Lincoln, 1986; Werkhoven, Snippe, \& Toet, 1992). In these studies, the task consists of determining whether the velocity of a stimulus is increasing or decreasing. It is then possible to determine a detection threshold as a function of a percentage of variation of velocity in the form of a Weber's law. This percentage is defined as a velocity ratio $\left(v_{\text {ratio }}\right)$ corresponding to the difference between final velocity and initial velocity with respect to average velocity, that is, $\left[\left(v_{\text {final }}-v_{\text {initial }}\right) \div v_{\text {average }}\right] \times 100$ (e.g., Babler \& Dannemiller, 1993; Calderone \& Kaiser, 1989; Regan et al., 1986). Although the results vary and are influenced by experimental conditions (see Brouwer et al., 2002, for a review), acceleration appears to be detectable with viewing time as short as $300 \mathrm{~ms}$ when the $v_{\text {ratio }}$ is as low as $20 \%-25 \%$ (e.g., Babler \& Dannemiller, 1993; Brouwer et al., 2002; Werkhoven et al., 1992).

The question that arises from these results is whether the processes involved in the perception of acceleration are also used in the timing of interceptive action. ${ }^{3}$ For one thing, in detection tasks, the information used to respond is qualitative ("there is acceleration" or "there is no acceleration"), whereas in interceptive actions, the required information for timing is necessarily quantitative. For another, the level of sensitivity required of the perceptual system in the detection task is relatively weak compared with the accuracy required in the timing of some interceptive actions that occurs around several milliseconds (e.g., Regan, 1997).

Werkhoven et al. (1992) suggested that the detection of acceleration evident in psychophysics experiments could be due to processes that compare the velocity of the stimulus at different instants rather than to a visual receptor permitting direct perception of acceleration (see also Brouwer et al., 2002). This suggests that there may be two independent mechanisms that depend on the task requirement: one for verbal-cognitive identification, the other for motor control. Making reference to the two visual pathways identified at the neurofunctional level (e.g., Goodale \& Milner, 1992; Keil, Holmes, Bennett, Davids, \& Smith, 2000), Dubrowski and Carnahan (2000) suggested that the information given by a moving stimulus could be used either in the ventral (or cognitive-visual) stream or in the dorsal (or motor-visual) stream with respect to the response to be produced (see also Tresilian, 1995, 1999b, for a discussion). In another study, Dubrowski and Carnahan (2002) showed that prior exposure to the interception of accelerated targets did not facilitate acceleration detection. This result is further evidence that the perception and especially the use of information about acceleration occur differently in verbal identification tasks and in interceptive action tasks. Nevertheless, although the psychophysical results say little about the information that is used in interceptive timing, the identification of acceleration could be used as a cue in this timing. Thus, the possible influence of acceleration detection on the timing of interceptive actions remains to be addressed.

\section{Synthesis}

From the review of the literature it appears that questions about information specifying acceleration and the regulation of intercep- tive actions are far from answered. Theoretically, a large number of actions could be timed on the basis of TTC1 information (e.g., tau) without need of information for acceleration (e.g., Bootsma et al., 1997; Lee et al., 1983; Tresilian, 1995). Nevertheless, there is limited empirical evidence to support this, and most existing studies provide qualitative data that are only compatible with the TTC1 hypothesis, not tests of quantitative predictions (Bootsma \& Oudejans, 1993; Dubrowski \& Carnahan, 2000, 2002; Kaiser \& Hecht, 1995; Lee et al., 1983, 1997; Port, Lee, Dassonville, \& Georgopoulos, 1997). In addition, recent work suggests that other variables could be used in certain situations (Lacquaniti et al., 1993; McIntyre et al., 2001; Tresilian, 1994a, 1994b) or for certain phases of movement (Michaels et al., 2001). Finally, psychophysical limits on the detection of acceleration have to be addressed. This ensemble of issues suggests that the use of TTC1 or TTC2 information for timing of interceptive actions still requires rigorous evaluation (Tresilian, 1999a).

The goal of the present study was to determine whether TTC1 or TTC2 information is used either to judge arrival or to intercept an accelerating or decelerating stimulus. In the first experiment, we used a PM task that consisted of presenting a horizontal moving stimulus that was occluded just before reaching a specified position. The observer was then required to make a simple response (e.g., press a button) coinciding temporally with the moving stimulus's arrival. This task, previously used by Rosenbaum (1975) and Kaiser and Hecht (1995), requires production of a response based on predictive information obtained before the occlusion of the stimulus. In this type of task, the use of TTC1 information should result in predictable overestimations of TTC when the stimulus accelerates and predictable underestimations when it decelerates, whereas the use of TTC2 information should not result in increased estimate errors with accelerated and decelerated trajectories. In order to clearly dissociate the predictions of TTC1 and TTC2 (Kaiser \& Hecht, 1995; Rosenbaum, 1975), we selected greater accelerations and longer occlusion times. In addition, because Schiff and Oldak (1990) have pointed out that errors and variability in responses in PM tasks are smaller for lateral motions than for direct approaches (see also Kaiser \& Mowafy, 1993; Yakimoff, Mateeff, Ehrenstein, \& Hohnsbein, 1993), lateral motion was used in order to minimize the variability in responses. Trajectories that were below and above the acceleration-detection threshold were used to address the question concerning the influence of a possible detection of acceleration in the estimation of TTC.

In the second experiment, we used an indirect interceptive task in which participants intercepted a moving stimulus using a ball

\footnotetext{
${ }^{3}$ Some recent studies have tried to test Chapman's (1968) hypothesis, according to which locomotor displacements required to catch a ball in flight could depend directly on the perception of the acceleration of the ball (e.g., Babler \& Dannemiller, 1993; Brouwer et al., 2002; P. McLeod, Reed, \& Dienes, 2001; Michaels \& Oudejans, 1992; Oudejans, Michaels, Bakker, \& Davids, 1999). According to these studies, the actor's strategy could consist of searching for, by his or her own appropriate movements, the vertical optical acceleration cancellation (OAC). Although the OAC hypothesis in the regulation of locomotion is very attractive, it cannot explain the timing of an effector in catching or hitting a ball. For this reason, we do not develop it in this article (for a discussion, see Benguigui, Ripoll, \& Broderick, 2002; P. McLeod et al., 2001).
} 
that had to be projected a distance of $2.20 \mathrm{~m}$. The purpose of this task was to require production of an action based on predictive information acquired before the arrival of the stimulus at the interception point, analogous to the PM task. Once the projectile was released, it could not be further controlled. This task was unlike "direct" interceptive actions such as catching or striking a ball, because continuous control of effector movement in the moments preceding contact was no longer possible. Consequently, as in a PM task, it was possible to predict participants' responses as a function of the temporal information they used. Specific and predictable errors should have been committed with the use of TTC1 information in projecting the ball too soon or too fast (underestimates) to intercept decelerating trajectories and too late or too slowly (overestimates) to intercept accelerating trajectories, whereas the use of TTC2 information should not result in increased estimate errors with accelerated and decelerated trajectories.

\section{Experiment 1}

\section{Method}

Participants. Eight male students (mean age $=24.3$ years) participated in this experiment. All participants were right-handed with normal or corrected-to-normal vision.

Apparatus. The apparatus consisted of a 400- $\times 7$-cm horizontal LED "runway." The anterior face of the runway was composed of 200 red LEDs spaced $2 \mathrm{~cm}$ apart. This separation corresponded to a $0.10^{\circ}$ angle at the left end of the runway between each LED from the point of observation and a $0.40^{\circ}$ angle at the right end of the runway. The successive illumination of the LEDs simulated an apparent movement of an object. The frequency with which the LEDs were illuminated depended on the velocity of the stimulus because the LEDs were positioned at a fixed interval. The illumination frequency just before occlusion ranged from 50 to $185 \mathrm{~Hz}$ in the experimental conditions. The stimulus moved horizontally left to right toward a target situated at the extreme end of the runway. The target was represented by two green LEDs, placed above and below the last LED. The illumination timing of the LEDs, trial onset, and data acquisition were synchronized on a $200-\mathrm{Hz}$ computer. The same system was previously used by Montagne, Fraisse, Ripoll, and Laurent (2000).

Task. A 96- $\times 230-\mathrm{cm}$ table was arranged perpendicular to the runway, so that the participants sat directly facing the target LED at a distance of $240 \mathrm{~cm}$ from it. The experiment took place in a normally illuminated room. The participants manually controlled two buttons situated $50 \mathrm{~cm}$ from each other. The left button, which was to be pressed with the left hand, initiated the movement of the moving stimulus. The interval between the left button press and the appearance of the stimulus varied randomly between 200 and $1,000 \mathrm{~ms}$.

The stimulus began its motion but disappeared before reaching the target. The occlusion was produced by the nonillumination of the LEDs situated in the "occlusion zone." The task consisted of estimating the moment of the stimulus' unseen arrival at the target by pressing the right button with the right hand at the moment the participant believed the stimulus reached the target. The time difference calculated by the computer between participant estimate of arrival time and real arrival time (i.e., the occlusion time) defined response accuracy (in milliseconds). Early responses were marked with a negative sign, whereas late responses were marked with a positive sign.

Procedure. After being informed by the experimenter of the test procedures, the participants were given several trials to become familiar with the task. During this familiarization period, the stimulus' velocity was 2.5 $\mathrm{m} / \mathrm{s}$ and two occlusion times (450 and $900 \mathrm{~ms}$ ) were used. The participants were given two blocks of 10 practice trials with corrective feedback about task procedures and qualitative knowledge of results $(\mathrm{KR})$ in the form "too early" (constant error $[\mathrm{CE}]<-100 \mathrm{~ms}$ ), "too late" (CE $>100 \mathrm{~ms}$ ), or "good response" $(-100 \mathrm{~ms}<\mathrm{CE}<100 \mathrm{~ms})$.

During the test, no feedback was given to the participants to ensure that only the basic ability of the visual system to extrapolate accelerated trajectories was tested. Eight different accelerations $(-2.45,-1.75,-1.05$, $\left.-0.35,0.35,1.05,1.75,2.45 \mathrm{~m} / \mathrm{s}^{2}\right)$ and eight occlusion times $(300,500$, $640,755,854,943,1,025,1,110 \mathrm{~ms}$ ) were used. The combination of the two independent variables led to 64 different trajectories. The starting position of the moving stimulus was always the same (i.e., $3.98 \mathrm{~m}$ from the target). As a consequence, the viewing time ranged from 0.6 to $1.4 \mathrm{~s}$ as a function of acceleration and occlusion time. A preliminary experiment had shown that the viewing time had no effect on extrapolation when it was above 0.24 s (Benguigui, 1997; see also Rosenbaum, 1975, for a similar result). The distances for which the stimulus was visible and occluded ranged respectively from 0.59 to $3.80 \mathrm{~m}$ and from 3.39 to $0.18 \mathrm{~m}$ as a function of acceleration and occlusion time.

The trials were randomly ordered in eight blocks of eight trials. The acceleration values were not known a priori by the participants. However, the trajectories selected were both above and below the 20\%-25\% threshold of $v_{\text {ratio }}$ for which acceleration is generally detectable. A control experiment was made to check the sensitivity of participants to the acceleration used (see below) with respect to the trajectories' $v_{\text {ratio }}$ in order to determine whether detection of acceleration could influence estimations in PM tasks.

\section{Data Analysis and Results}

First analysis: Using TTC1 or TTC2. The goal of the first analysis was to determine whether the temporal estimates of the arrival of the stimulus occurred on the basis of TTC 2 or TTC1. We calculated TTC1 and TTC2 at the moment of occlusion on the basis of linear motion (referred to the runway) and angular motion (referred to the observer) ${ }^{4}$ :

TTC1 for linear motion was calculated as $\operatorname{TTC} 1(x)=x \div \dot{x}$; TTC2 for linear motion was calculated as $\operatorname{TTC} 2(x)=(-\dot{x}+$ $\left.\sqrt{\dot{x}^{2}-2 \cdot \ddot{x} \cdot x}\right) \div \ddot{x} ;^{5}$

TTC1 for angular motion was calculated as $\operatorname{TTC} 1(\theta)=\theta \div \dot{\theta}$; and

TTC2 for angular motion was calculated as $\operatorname{TTC} 2(\theta)=(-\dot{\theta}+$ $\left.\sqrt{\dot{\theta}^{2}-2 \cdot \ddot{\theta} \cdot \theta}\right) \div \ddot{\theta}$

where $x$ is the distance between the stimulus at occlusion and the arrival point, $\dot{x}$ is the instantaneous linear velocity, $\ddot{x}$ is the instan-

\footnotetext{
${ }^{4}$ Angular and linear object motion are exactly proportional only when objects rotate around the point of observation. The difference between an object with constant velocity and constant optical velocity is not an issue for short movements in a fronto-parallel plane viewed from some distance. This is in contrast to our experiment in which the runway was rectilinear, the motion displacement was large, and occlusions occurred at different positions $(0.18 \mathrm{~m}$ from the target for the shorter occlusion to $3.40 \mathrm{~m}$ from the target for the longer occlusion). For example, the angular velocity of a stimulus moving at a constant linear velocity of $1 \mathrm{~m} / \mathrm{s}$ would be $6.37^{\circ} / \mathrm{s}$ at the beginning of the track and $23.87 \%$ at the end of the track, a $375 \%$ increase in the velocity from the beginning to the end of the track, corresponding to a mean angular acceleration of $4.38 \% \mathrm{~s}^{2}$.

${ }^{5}$ Note that in this case, TTC2 $(x)$ is equal to the real TTC-that is, TTC $(x)$ - because the stimulus's accelerations were constant.
} 
taneous linear acceleration, $\theta$ is the angle formed by the stimulus at occlusion and the target with respect to the participant, $\dot{\theta}$ is the instantaneous angular velocity of the closure of this angle, and $\ddot{\theta}$ is the angular acceleration of the closure of this angle (see Figure 2).

Four regression analyses were performed for each participant, with the time estimate as the dependent variable and $\operatorname{TTC} 1(x)$, $\operatorname{TTC} 2(x), \operatorname{TTC} 1(\theta)$, and $\operatorname{TTC} 2(\theta)$ at the moment of occlusion as the independent variables. All the correlations were significant, which follows from the fact that significant correlations exist between the four predictors (see Table 1). However, for 7 participants, TTC1 $(x)$ was a better predictor than the others (see the $r^{2}$ in the top half of Table 2 and Figure 3 ). For Participant 4, TTC $(\theta)$ was the better predictor. An analysis of variance (ANOVA) with the four predictors-TTC1 $(x), \quad \operatorname{TTC} 2(x), \quad \operatorname{TTC} 1(\theta)$, and $\operatorname{TTC} 2(\theta)$ - as independent variables performed on the $r^{2}$ for each participant transformed to a Fisher $z$ showed a significant effect, $F(3,21)=46.93, p<.05$. A Newman-Keuls post hoc analysis showed that $r^{2}$ for $\operatorname{TTC} 1(x)$ and $\operatorname{TTC} 1(\theta)$ were significantly higher $(p<.05)$ than $r^{2}$ for $\operatorname{TTC} 2(x)$ and $\operatorname{TTC} 2(\theta)$. No significant difference appeared between $\operatorname{TTC} 1(x)$ and $\operatorname{TTC} 1(\theta)$.

The dispersion of the data for either $\operatorname{TTC} 1(x)$ or $\operatorname{TTC} 1(\theta)$ as a predictor was not negligible with a mean $r^{2}$ of .81 and .79, respectively (see the top half of Table 2). However, this variability is undoubtedly connected to the characteristics of the PM task, specifically to the temporal extrapolation required, which is a source of response variability (for discussion, see, e.g., Bootsma, Marteniuk, \& MacKenzie, 1991; DeLucia \& Lidell, 1998; Kaiser \& Mowafy, 1993; Tresilian, 1994b, 1995). Note that the dispersion increased with the occlusion time (see Figure 3), which is also a classical result in PM tasks.

At this stage, our results show that time estimations were made on the basis of first-order information. Although it is not possible to make a distinction between $\operatorname{TTC} 1(x)$ and $\operatorname{TTC} 1(\theta)$ on the basis of $r^{2}$, an examination of the slopes of the linear regression enables us to distinguish the possible utilization of one from the other in this task. For TTC1 $(x)$, the slopes ranged from 0.66 to 1.03 (0.86 over all participants; see the top half of Table 2) and are quite comparable to the values found in a number of studies using PM tasks (e.g., 0.74 for Carel, 1961; 0.61 for Schiff \& Detwiler, 1979; 0.58 for R. W. McLeod \& Ross, 1983; 0.73 for expert drivers and 0.65 for novices for Cavallo \& Laurent, 1988; 0.84 for Kaiser \& Mowafy, 1993, Experiment 2; 1.00 for Yakimoff et al., 1993; 0.84 for men and 0.65 for women for Caird \& Hancock, 1994; 0.67 for Sidaway, Fairweather, Sekiya, \& McNitt-Gray, 1996). The present slopes demonstrate a tendency toward slight overestimations of $\operatorname{TTC}(x)$ for short occlusions $(<1 \mathrm{~s})$ and slight underestimation for

Table 1

Coefficients of Regression Between the Four Predictors in Experiment 1

\begin{tabular}{lcccc}
\hline Predictor & TTC1 $(x)$ & TTC2 $(x)$ & TTC1 $(\theta)$ & TTC2 $(\theta)$ \\
\hline TTC1 $(x)$ & - & & & \\
TTC2 $(x)$ & .53 & - & & \\
TTC1 $(\theta)$ & .98 & .46 & - & \\
TTC2 $(\theta)$ & .94 & .73 & .90 & - \\
\hline
\end{tabular}

Note. All correlations were significant. TTC $=$ time to contact. occlusions around $1 \mathrm{~s}$, consistent with the characteristics of PM tasks (for discussion, see, e.g., Caird \& Hancock, 1994; Schiff \& Oldak, 1990; Yakimoff et al., 1993). For TTC1( $\theta)$, on the other hand, the slopes range from 0.30 to 0.48 ( 0.40 over all participants; see the top half of Table 2), meaning that participants' time estimates would be half than the predicted time. The predictions range up to $3,958 \mathrm{~ms}$, whereas participants' estimations would range up to 2,159 $\mathrm{ms}$ (see Figure $3 \mathrm{C}$ as an illustration). Note that predictions with $\operatorname{TTC} 1(x)$ range up to $1,952 \mathrm{~ms}$. This observation eliminates the possible use of $\operatorname{TTC} 1(\theta)$ to estimate the arrival time of the moving object.

Second analysis: Using TTC1 or TTC2 when the $\mathrm{v}_{\text {ratio }}$ is above the detection threshold. To test the influence of the detection of acceleration on temporal estimates, we performed a similar analysis in which we excluded trajectories with $v_{\text {ratios }}$ below the detection threshold. For this purpose, a complementary experiment was done to identify the exact threshold under present testing conditions.

The visual system sensitivity in acceleration detection was assessed in a two-alternative forced-choice task in which the 8 participants verbally determined in 125 trials whether the presented trajectories were accelerated or decelerated. The participants were positioned in the same condition as in the first experiment. Twenty-five different trajectories were used with $v_{\text {ratios }}$ from $-60 \%$ to $60 \%$, with a step of $4 \%$ between $-40 \%$ and $40 \%$ and a step of $10 \%$ under $-40 \%$ and above $40 \%$. Note that the $v_{\text {ratios }}$ were calculated on the basis of linear motion (the $v_{\text {ratios }}$ calculated on the basis of the angular velocities yielded only positive values from $37 \%$ to $128 \%$ of variation). The velocities and accelerations of the trajectories were selected to be in the same range as the velocities and the accelerations used in the PM task. The viewing time for all trajectories was identical (1,000 ms). To determine the $v_{\text {ratio }}$ threshold for each participant, we performed a logit fit of the psychometric function generated by the response as a function of $v_{\text {ratio }}$. For each participant, the percentage of responses in which the participant detected acceleration was calculated. The thresholds of discrimination for acceleration and deceleration were defined in calculating the $v_{\text {ratios }}$ for the point at which the fitted psychometric function crosses $25 \%$ for the decelerated trajectories (negative $v_{\text {ratio }}$ ) and $75 \%$ for the accelerated trajectories (positive $v_{\text {ratio }}$ ). Results show that the mean $v_{\text {ratio }}$ threshold for detecting deceleration and acceleration were respectively $-21.1 \%$ and $15.7 \%$ (absolute mean $=18.36 \%$, ranging for the 8 participants from $10.7 \%$ to $30.0 \%$ ).

This result means two things. First, it confirms that discrimination of acceleration is based on information about linear velocity and not only on angular velocity, as in the latter case participants should have perceived only accelerations. Second, the obtained values were in line with the previous results of Babler and Dannemiller (1993), who found $v_{\text {ratio }}$ thresholds of $-17.0 \%$ and $20.4 \%$ and $-21.6 \%$ and $16.4 \%$, in two different experiments respectively, as well as those of Werkhoven et al. $(1992 ; 17 \%)$ and Brouwer et al. $(2002 ; 20-25 \%)$.

To test whether the acceleration detection influenced the temporal estimates, we excluded PM trajectories that had a threshold between $-30 \%$ and $30 \%$, to ensure that acceleration was above the detection threshold for all participants. The $v_{\text {ratios }}$ for the 64 test 
Table 2

Results of the Different Regression Analyses Between TTC1(x), TTC2(x), TTC1( $\theta)$, and TTC2( $\theta)$ and Participants' TTC Estimates for All Trajectories and for Trajectories With an Absolute $\mathrm{v}_{\text {ratio }}$ Value Greater Than $30 \%$ in Experiment 1

\begin{tabular}{|c|c|c|c|c|c|c|c|c|c|c|}
\hline \multirow[b]{2}{*}{ Part. } & \multicolumn{3}{|c|}{ Estimated TTC-TTC1 $(x)$} & \multicolumn{3}{|c|}{ Estimated TTC-TTC2 $(x)$} & \multicolumn{3}{|c|}{ Estimated TTC-TTC1 $(\theta)$} & Estimated TTC-TTC2 $(\theta)$ \\
\hline & Slope & Intercept & $r^{2}$ & Slope & Intercept & $r^{2}$ & Slope & Intercept & $r^{2}$ & Intercept \\
\hline
\end{tabular}

\begin{tabular}{|c|c|c|c|c|c|c|c|c|c|c|c|c|}
\hline \multicolumn{13}{|c|}{ All trajectories } \\
\hline 1 & 0.85 & 59 & .84 & 0.92 & 13 & .43 & 0.39 & 257 & .80 & 1.03 & -72 & .81 \\
\hline 2 & 1.03 & -15 & .93 & 1.07 & -38 & .44 & 0.48 & 219 & .91 & 1.22 & -149 & .85 \\
\hline 3 & 1.02 & 13 & .88 & 0.97 & 64 & .34 & 0.48 & 244 & .87 & 1.18 & -99 & .77 \\
\hline 4 & 0.88 & -85 & .85 & 0.72 & 41 & .25 & 0.42 & 100 & .89 & 0.98 & -154 & .69 \\
\hline 5 & 0.78 & 216 & .75 & 0.86 & 156 & .41 & 0.35 & 404 & .70 & 0.94 & 95 & .73 \\
\hline 6 & 0.66 & 135 & .77 & 0.76 & 65 & .44 & 0.30 & 297 & .71 & 0.80 & 33 & .74 \\
\hline 7 & 0.89 & 116 & .85 & 0.97 & 57 & .45 & 0.41 & 317 & .83 & 1.06 & -11 & .80 \\
\hline 8 & 0.75 & 59 & .64 & 0.76 & 56 & .29 & 0.35 & 226 & .63 & 0.87 & -23 & .55 \\
\hline$M$ & 0.86 & 62 & .81 & 0.88 & 51 & .38 & 0.40 & 258 & .79 & 1.01 & -47 & \\
\hline$S D$ & 0.13 & 94 & .09 & 0.12 & 54 & .08 & 0.06 & 88 & .10 & 0.14 & 88 & .09 \\
\hline
\end{tabular}

\begin{tabular}{|c|c|c|c|c|c|c|c|c|c|c|c|c|}
\hline 1 & 0.84 & 62 & .83 & 0.97 & -14 & .38 & 0.38 & 249 & .80 & 1.07 & -99 & .80 \\
\hline 2 & 1.06 & -30 & .95 & 1.26 & -152 & .46 & 0.49 & 202 & .93 & 1.35 & -227 & .91 \\
\hline 3 & 1.05 & -12 & .92 & 1.12 & -36 & .36 & 0.49 & 218 & .91 & 1.31 & -182 & .83 \\
\hline 4 & 0.92 & -97 & .90 & 0.88 & -43 & .28 & 0.43 & 95 & .92 & 1.12 & -226 & .78 \\
\hline 5 & 0.75 & 202 & .83 & 0.83 & 162 & .35 & 0.34 & 375 & .78 & 0.94 & 73 & .77 \\
\hline 6 & 0.65 & 134 & .77 & 0.82 & 20 & .42 & 0.29 & 287 & .71 & 0.84 & 5 & .75 \\
\hline 7 & 0.88 & 113 & .86 & 1.05 & 12 & .41 & 0.41 & 305 & .85 & 1.12 & -48 & .81 \\
\hline 8 & 0.78 & 49 & .71 & 0.92 & -29 & .33 & 0.36 & 222 & .69 & 0.98 & -81 & .65 \\
\hline$M$ & 0.87 & 53 & .84 & 0.98 & -10 & .37 & 0.40 & 244 & .82 & 1.09 & -98 & .79 \\
\hline$S D$ & 0.14 & 97 & .08 & 0.15 & 87 & .06 & 0.07 & 83 & .09 & 0.17 & 109 & .07 \\
\hline
\end{tabular}

Note. $\quad$ TTC $=$ time to contact; Part. = participant.

stimuli varied from $-126 \%$ to $173 \%$. Of these, 19 (29.7\%) were below the thresholds. ${ }^{6}$ The four regression analyses were then performed on the $45(70.3 \%)$ remaining suprathreshold trajectories (see the bottom half of Table 2 and Figure 3, full circle). As in the previous analyses, $\operatorname{TTC} 1(x)$ was the better predictor for 7 participants, whereas $\operatorname{TTC} 1(\theta)$ was the better predictor for Participant 4. An ANOVA with the four predictors-TTC1 $(x), \operatorname{TTC} 2(x)$, $\operatorname{TTC} 1(\theta)$, and $\operatorname{TTC} 2(\theta)$ - as independent variables performed on the $r^{2}$ for each participant transformed to a Fisher $z$ showed a significant effect, $F(3,21)=57.02, p<.05$. A Newman-Keuls post hoc analysis showed that $r^{2}$ values for TTC1( $\left.x\right)$ were significantly different from $r^{2}$ values for $\operatorname{TTC} 2(x)$ and $\operatorname{TTC} 2(\theta)$ and that $r^{2}$ values for $\operatorname{TTC} 1(\theta)$ were significantly different from $r^{2}$ values for TTC2 $(x)$. Examination of the slopes showed the same difference between $\operatorname{TTC} 1(x)$ and $\operatorname{TTC} 1(\theta)$ as in the previous experiment (0.87 vs. 0.40$)$. Thus, even when acceleration was detectable, TTC2 information was not used to estimate TTC in this PM task and $\operatorname{TTC} 1(x)$ was the better predictor of time estimations.

Third analysis: Errors with accelerated trajectories. The goal of the third analysis was to show that a $\operatorname{TTC} 1(x)$ strategy induces errors in estimates of arrival time as a function of the occlusion time and the value of acceleration. The CEs were compared in an ANOVA for the independent variable occlusion at eight levels $(300,500,640,755,854,943,1,025,1,110 \mathrm{~ms})$ and for the independent variable acceleration at eight levels $(-2.45,-1.75$, $-1.05,-0.35,0.35,1.05,1.75,2.45 \mathrm{~m} / \mathrm{s}^{2}$ ). The analysis revealed a main effect of occlusion, $F(7,448)=3.44, p<.05$; a main effect of acceleration, $F(7,448)=124.92, p<.05$; and significant Occlusion $\times$ Acceleration interaction, $F(49,448)=3.29, p<.05$ (see Figure 4). These effects demonstrate that the errors in extrapolations increase as both occlusion time and acceleration increase. This provides additional evidence for the use of $\operatorname{TTC} 1(x)$ information, because the differential among $\operatorname{TTC} 1(x)$ and $\operatorname{TTC}(x)$ increases when $\operatorname{TTC}(x)$ (i.e., the occlusion time in the present experiment) and the values of accelerations increase (see Figure 1).

The distributions of overestimations and underestimations due to acceleration and deceleration for each occlusion condition are also influenced by the time of occlusion. There is slightly more

\footnotetext{
${ }^{6}$ In the first experiment, the viewing time for the trajectories ranged from 0.6 to $1.4 \mathrm{~s}$. The $v_{\text {ratios }}$ for these trajectories were calculated with two different procedures. First, the viewing time was not taken into account and the $v_{\text {ratios }}$ were calculated with the following formula: $v_{\text {ratio }}=\left[\left(v_{\text {final }}-\right.\right.$ $\left.\left.v_{\text {initial }}\right) \div v_{\text {average }}\right] \times 100$. Second, the viewing time was integrated in a normalization of the $v_{\text {ratio }}$. As the $v_{\text {ratios }}$ in the complementary experiment were $1 \mathrm{~s}$, the $v_{\text {ratios }}$ were calculated on the basis of a variation of velocity per second with the following formula: $v_{\text {ratio }}=\left\{\left[\left(v_{\text {final }}-v_{\text {initial }}\right) \div v_{\text {average }}\right]\right.$ $\times$ viewing time $\} \times 100$. Of 64 experimental trajectories, 19 were above the threshold of $30 \%$ using the first calculation and 16 using the second. We selected the first calculation because it excluded more of trajectories, to be sure that in our new analysis all the trajectories really were above the detection threshold.
} 

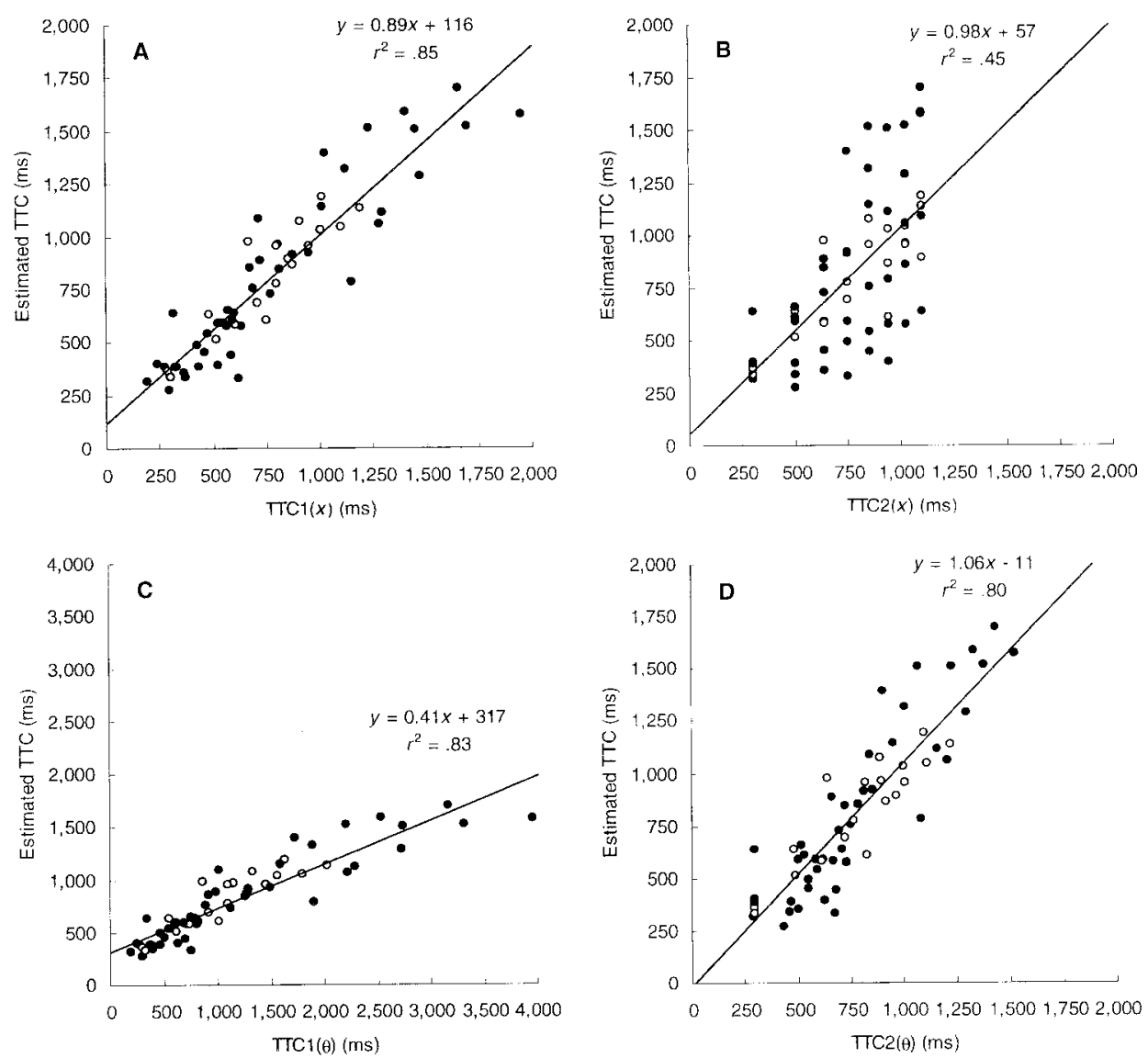

Figure 3. Regression analyses between $\operatorname{TTC} 1(x), \operatorname{TTC} 2(x), \operatorname{TTC} 1(\theta)$, and $\operatorname{TTC} 2(\theta)$ at the moment of occlusion (A, B , C, and D, respectively) and TTC estimates for Participant 7 (who produced typical values) in Experiment 1. Note that the scale for Panel $\mathrm{C}$ is different from that of the three others. The full circles correspond to the trajectories with an absolute value of $v_{\text {ratio }}$ greater than $30 \%$. The empty circles correspond to the trajectories with $v_{\text {ratio }}$ less than $30 \%$. TTC $=$ time to contact; $x=$ the distance between the stimulus at occlusion and the arrival point; $\theta=$ the angle formed by the stimulus at occlusion and the target with respect to the participant.

overestimation at the shortest occlusion (300 ms) and slightly more underestimation for occlusions around $1 \mathrm{~s}(854,943,1,025,1,100$ $\mathrm{ms})$. These tendencies are linked to the characteristics of PM tasks in which overestimations are observed for short occlusions and underestimations for long occlusions (e.g., Caird \& Hancock, 1994).

Fourth analysis: Predicting errors. To confirm the TTC1 $(x)$ hypothesis, we sought to determine whether the CEs by the participants were quantitatively associated with the use of a TTC1( $x)$ strategy. For this, the theoretical errors corresponding to the use of $\operatorname{TTC} 1(x)$ were calculated by taking the difference between $\operatorname{TTC} 1(x)$ and TTC $(x)$ at the moment of the stimulus's occlusion (see Figure 5 for an illustration). The theoretical predictions ranged from $-500 \mathrm{~ms}$ (for the trajectory with the longest occlusion and the highest deceleration) to $852 \mathrm{~ms}$ (for the trajectory with the longest occlusion and the highest acceleration). They were then compared with CEs by using a regression analysis. The analyses yield regression lines that confirm the $\operatorname{TTC} 1(x)$ hypothesis (see Table 3, left half, and Figure 6 as an illustration for Participant 7). Within the variability inherent in PM tasks, the individual coeffi- cients of regression that vary between .45 and .88 (mean $\left.r^{2}=.68\right)$ can be considered rather high. The slopes of the regression lines, which range from 0.60 to 1.18 for individual participants (mean $=$ 0.91), show that the CEs correspond quantitatively to the predicted errors. A second analysis included only the trajectories with an absolute value of $v_{\text {ratio }}$ greater than $30.0 \%$ ( $70.3 \%$ of the trajectories). The results of this analysis are very close to the first analysis (Table 3, right half; Figure 6, full circle) and suggest that the CEs by the participants are not influenced (minimized) by suprathreshold acceleration. This means that even when acceleration is psychophysically detectable, participants do not use this information to minimize their errors.

\section{Discussion}

The analyses show that TTC1 $(x)$ better accounts for estimates of TTC than do the other temporal variables. Our lateral PM task also supplements the head-on motion findings of Kaiser and Hecht (1995), as our participants overestimated TTC when the stimulus accelerated and underestimated TTC when it decelerated. These 


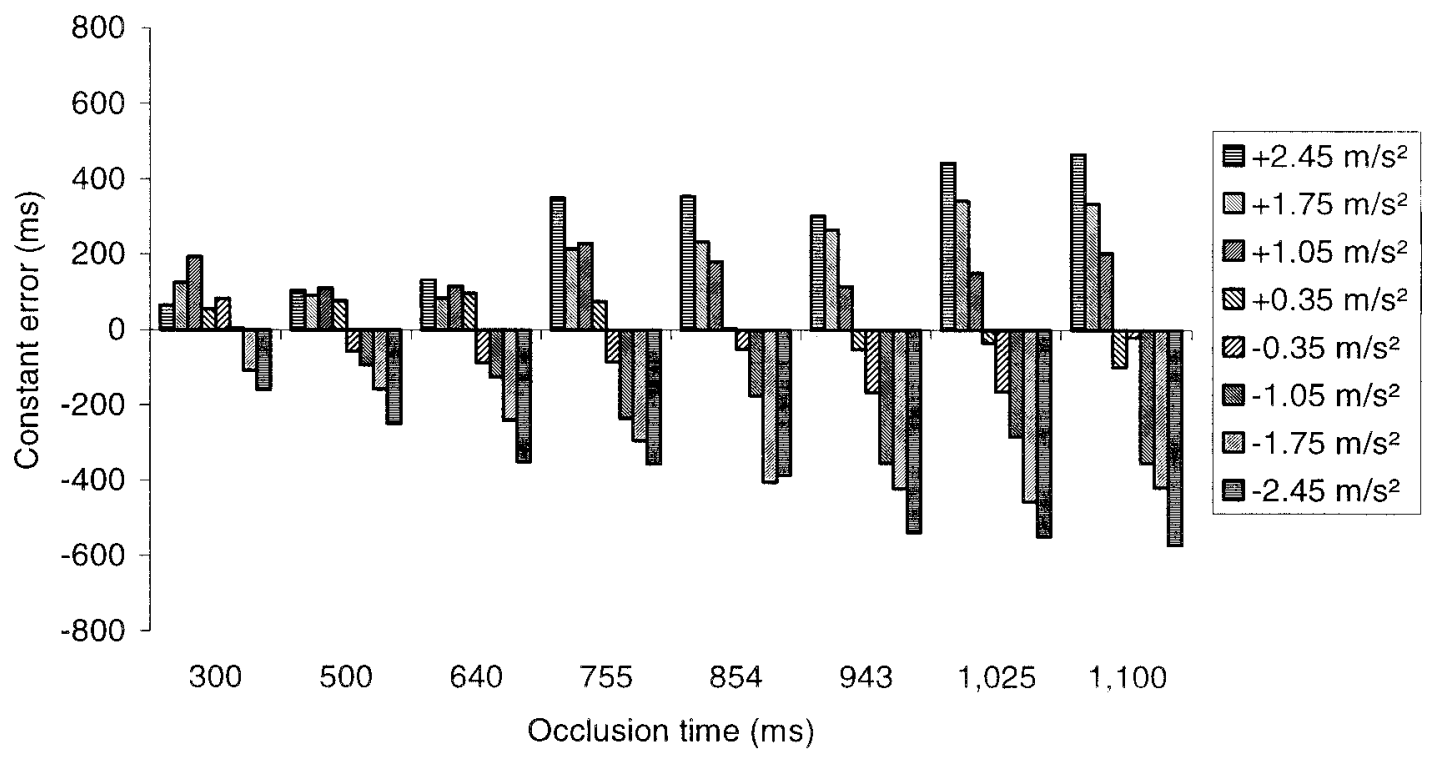

Figure 4. Constant errors as a function of occlusion time and acceleration values in Experiment 1. The errors increase with the increase of both occlusion and acceleration. Positive errors correspond to overestimations of TTC and negative errors to underestimations. TTC $=$ time to contact.

results suggest that the absence of a difference between acceleration and constant velocity conditions observed by Rosenbaum (1975) was most likely due to his use of low values of acceleration.

These conclusions are confirmed by the fourth analysis, which shows that the CEs correspond quantitatively to errors predicted by a $\operatorname{TTC} 1(x)$ strategy. This finding clearly demonstrates that participants do not use information about the acceleration of the stimulus to estimate TTC. This is true even when acceleration is psychophysically detectable, because the correlations between predicted and CEs were not different when subthreshold trajectories were excluded. This confirms the hypothesis that acceleration detection is not implicated in a task requiring determination of TTC (Dubrowski \& Carnahan, 2000, 2002). It is also in accordance with the suggestion of Werkhoven et al. (1992) that human detection of velocity modulation is not directly based on acceleration information but is rather based on a perception of the differences between velocities at distinct instants.

These results also confirm that the findings of Lacquaniti and his colleagues (Lacquaniti et al., 1993; Lacquaniti \& Maioli, 1989a, 1989b; McIntyre et al., 2001) are specific to situations in which acceleration is due to gravity. If an internal model of acceleration was involved in time estimations, the errors should have been less than they were. The participants, knowing that the stimulus was accelerated or decelerated, should have pressed the key before or after, respectively, they actually did.

A question arising from our results concerns the generalization of these data to interceptive actions. Indeed, PM tasks and interceptive tasks do not appear to be completely equivalent (see Tresilian, 1995, for a discussion). The first difference is that the responses in PM tasks are made when the stimulus is not visible (Bootsma et al., 1991; Tresilian, 1995). The second difference is that the arrival point is fixed and not chosen by the actor as it is in the case of interceptive tasks. Nevertheless, it can be hypothesized that people rely on $\operatorname{TTC} 1(x)$ information in both cases, and that the principal difference between them is how this temporal information is used. A second experiment was conducted in order to confirm the use of TTC1 $(x)$ information with accelerated trajectories in a more ecological task.

\section{Experiment 2}

The goal of the second experiment was to determine whether people use information specifying $\operatorname{TTC} 1(x)$ and ignore acceleration for interceptive actions in the same way they do for the PM task. We used an indirect interceptive task consisting of having a participant project a ball to intercept the moving stimulus a distance of $2.20 \mathrm{~m}$. In this task, the action was completed when the stimulus was visible and the interceptive point itself was chosen by the participants. These two factors added degrees of freedom which are typically present in ecological situations (Bootsma et al., 1991; Tresilian, 1995). However, like the preceding PM task, this task requires use of predictive information. Once the projectile is released, no further regulation of the action is possible, in contrast to catching or striking tasks. We used this task in order to test whether $\operatorname{TTC} 1(x)$ information is used in interceptive timing and whether this would yield quantifiable interception errors.

In this experiment we also sought to determine which information variable might be used to initiate the interceptive movement. We were interested in testing the conclusions of Michaels et al. (2001) concerning whether different sources of information are used in initiation, on the one hand, and timing, on the other. With this goal, an analysis of the same type used by Michaels et al. was performed in order to test the different variables that could account for initiating the interceptive movement. We thus tested three temporal variables- $\operatorname{TTC} 2(x), \operatorname{TTC} 1(x)$, and TTC $1(x)$, the latter being the variation of $\operatorname{TTC} 1(x)$ across time-and three optical variables $-\theta$ (the angle formed by the stimulus and the point of 


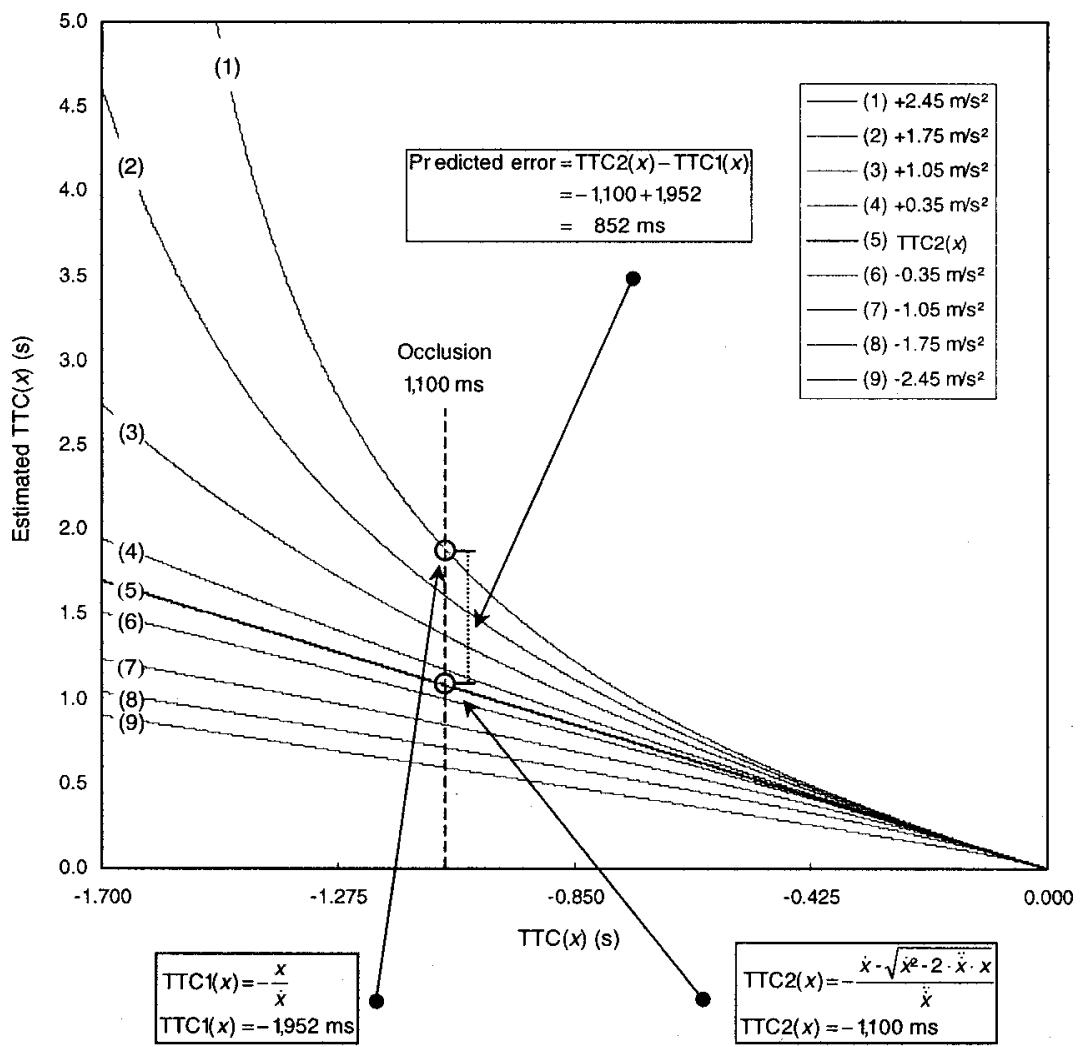

Figure 5. Evolution of $\operatorname{TTC} 2(x)$ and $\operatorname{TTC} 1(x)$ as a function of $\operatorname{TTC}(x)$ for the experimental trajectories in Experiment $1 ; x$ corresponds to the distance between the stimulus and the target. Note that $\operatorname{TTC} 2(x)$ is equal to $\operatorname{TTC}(x)$ because the accelerations are constant. In the example presented for the error calculation, the stimulus's acceleration is $2.45 \mathrm{~m} / \mathrm{s}^{2}$ and the occlusion time is $1,100 \mathrm{~ms}$. The expected error is $852 \mathrm{~ms}$. TTC = time to contact.

interception with respect to the participant's observation point), $\dot{\theta}$ (the velocity of constriction of the angle $\theta$ ), and $\ddot{\theta}$ (the acceleration in the constriction of this angle; see Figure 2).

Table 3

Results of the Different Regression Analyses Between Predicted Errors and Measured Errors for Each Participant in Experiment 1

\begin{tabular}{|c|c|c|c|c|c|c|}
\hline \multirow[b]{2}{*}{ Participant } & \multicolumn{3}{|c|}{$\begin{array}{l}\text { AllTr: constant errors- } \\
\text { predicted errors }\end{array}$} & \multicolumn{3}{|c|}{$\begin{array}{l}\text { Tr30: constant errors- } \\
\text { predicted errors }\end{array}$} \\
\hline & Slope & Intercept & $r^{2}$ & Slope & Intercept & $r^{2}$ \\
\hline 1 & 0.86 & -53 & .69 & 0.85 & -61 & .70 \\
\hline 2 & 1.10 & 9 & .88 & 1.09 & 12 & .91 \\
\hline 3 & 1.18 & 31 & .85 & 1.17 & 25 & .89 \\
\hline 4 & 1.09 & -181 & .80 & 1.07 & -160 & .85 \\
\hline 5 & 0.75 & 45 & .55 & 0.77 & 16 & .66 \\
\hline 6 & 0.60 & -124 & .50 & 0.58 & -126 & .52 \\
\hline 7 & 0.88 & 31 & .72 & 0.88 & 25 & .74 \\
\hline 8 & 0.79 & 132 & .45 & 0.78 & -112 & .53 \\
\hline$M$ & 0.91 & -47 & .68 & 0.90 & -48 & .73 \\
\hline$S D$ & 0.20 & 89 & .16 & 0.19 & 72 & .14 \\
\hline
\end{tabular}

Note. $\mathrm{AllTr}=$ all trajectories; $\operatorname{Tr} 30=$ trajectories with an absolute $v_{\text {ratio }}$ greater than $30 \%$.

\section{Method}

Participants. $\quad$ Eight male students (mean age $=22.8$ years) participated in this experiment. All participants were right-handed with normal or corrected-to-normal vision.

Apparatus and task. For this experiment, the apparatus used in the first experiment was adapted so that participants could intercept the stimulus by rolling a tennis ball, using a horizontal extension of the forearm, along the top of a table $(230 \times 96 \mathrm{~cm})$ placed perpendicular to the end of the LED runway. The target end of the LED runway was placed above a 96- $X$ $10-\mathrm{cm}$ configuration of 24 strain gauges. We call this configuration the "interception zone." The 24 strain gauges, each $4-\mathrm{cm}$ wide, were placed vertically at the end of a table, and they registered the moment and the position of ball contact. The 24 strain gauges allowed determination of 48 positions in intervals of $2 \mathrm{~cm}$ each (when 2 strain gauges were touched by the ball, the position registered corresponded to the midpoint between the 2 strain gauges). Each position specified by the gauges was aligned with a specific LED. The position and the moment of ball contact with strain gauge were compared with the motion of the stimulus (LED illumination) to give the errors of spatial and temporal precision of the interception of the stimulus by the ball (see Figure 7).

To initiate a trial, the participants had to place the ball on a switch $(5-\mathrm{cm}$ diameter) located in front of them and $220 \mathrm{~cm}$ from the LED runway. The contact created by the ball triggered the stimulus motion after a variable delay of 40-400 ms. The participants were instructed to keep the ball on the switch up to the moment of initiating their propulsion movement. The beginning of the movement was recorded by interruption of ball-switch 


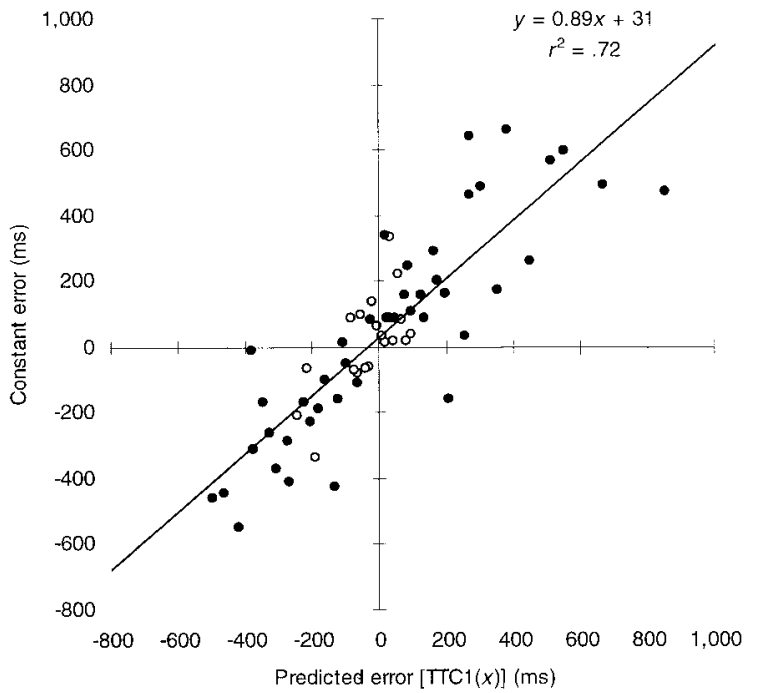

Figure 6. Constant errors as a function of predicted errors for Participant 7 (who produced typical values) in Experiment 1. The full circles correspond to the trajectories with an absolute value of $v_{\text {ratio }}$ greater than $30 \%$. The empty circles correspond to the trajectories with $v_{\text {ratios }}$ less than $30 \%$.

contact. The moment of release of the ball from the hand was recorded by a microswitch placed on the participant's thumb. The participants were instructed to project the ball in order to intercept the moving stimulus anywhere within the interception zone.

Procedure. After being informed of the test procedure, participants received a period of practice. This practice period consisted of two blocks of 10 trials. Immediately after each trial, participants were given KR of the spatial accuracy of their interception by the simultaneous illumination of the LED(s) associated with the gauge(s) they had hit with the ball and the LED associated with the stimulus at the moment the ball hit the gauge(s). The velocity of the stimulus used for the practice period was $2.5 \mathrm{~m} / \mathrm{s}$ and the total time of presentation $1.294 \mathrm{~s}$.

During the course of the test, nine accelerations were used: $-2.4,-1.8$, $-1.2,-0.6,0,0.6,1.2,1.8,2.4 \mathrm{~m} / \mathrm{s}^{2}$. The initial velocities of the trajectories ranged from 4.40 to $2.16 \mathrm{~m} / \mathrm{s}$. The $v_{\text {ratio }} \mathrm{s}$ calculated for the nine trajectories from the appearance of the stimulus to its arrival in the interception zone were, respectively, $-68,-51,-34,-17,0,17,34,51$, $68 \%$. For six of the nine trajectories, the $v_{\text {ratios }}$ were greater than the detection threshold identified in the control experiment.

In all conditions, the time of presentation of the stimulus before the arrival in the interception zone was $932 \mathrm{~ms}$. The mean frequency of LEDs' illumination was $164 \mathrm{~Hz}$ (with variation from 108 to 220 $\mathrm{Hz}$ as a function of the speed of the stimulus). The participants had two blocks of 27 trials, presented in a counterbalanced order. Within each block, the different trajectories were presented in random order. During the test, no feedback was furnished to the participants and the final part of the trajectory corresponding to the movement of the stimulus in the interception zone was occluded so that the participants would not know their errors with respect to the stimulus's acceleration. $^{7}$

Analysis of the errors. In this predictive task, the hypothesis of a $\operatorname{TTC} 1(x)$ strategy would be reflected by errors in the interceptive actions. We first made a qualitative analysis of the errors to verify our hypothesis that, for decelerating trajectories, the participants would project the ball too early (or too quickly; negative errors indicate overestimates of TTC $[x]$ ), whereas for the accelerating trajectories, they would project the ball too late (or slowly; positive errors indicate underestimates of TTC $[x]$ ). Second, we performed a quantitative analysis following the logic of Experiment 1. We thus sought to show that it is possible to predict quantitatively the errors committed by the participants.

The temporal errors were calculated by noting the positions of the stimulus and ball at the moment the ball contacted the strain gauges at the end of the table. The temporal error (in milliseconds) corresponded therefore to the time between the ball's contact with the strain gauges and the amount of time the stimulus would have needed to be at the position of the ball's contact, either less or more. If the stimulus had already passed the point where the ball hit the strain gauge, the sign of the error was marked with a positive sign. If the stimulus had not yet reached the point where the ball hit the strain gauge, the error was marked as negative (see Figure 7). The accuracy of the time measure, with the $2-\mathrm{cm}$ space between the strain gauges, was within $\pm 9 \mathrm{~ms}$ for the different trajectories and the different points of interception. The temporal errors were analyzed in order to obtain a $\mathrm{CE}$, that is, the algebraic mean over all the signed errors for each participant and for each acceleration condition.

\section{Results and Discussion}

Qualitative test of the TTC1(x) hypothesis. A repeated measures ANOVA was performed on CE, with acceleration as an independent variable $(-2.4,-1.8,-1.2,-0.6,0,0.6,1.2,1.8$, $\left.2.4 \mathrm{~m} / \mathrm{s}^{2}\right)$. Results revealed a main effect of acceleration, $F(8$, $56)=101.35, p<.05$ (see Figure 8 ). The participants committed negative errors, that is, $\operatorname{TTC}(x)$ overestimates, for the decelerating trajectories and positive errors, that is $\operatorname{TTC}(x)$ underestimates, for the accelerating trajectories. Furthermore, these estimation errors were proportional to the values of acceleration. These results support the hypothesis that participants do not use information about stimulus acceleration. It can be suggested that at a certain point, $\operatorname{TTC} 1(x)$ information is used to estimate TTC $(x)$, leading to respective overestimates for decelerations and underestimates for accelerations.

Quantitative test of the TTC1(x) hypothesis for movement initiation. Following the same logic of Experiment 1, we sought to demonstrate that it is possible to quantitatively predict the participants' errors. First, we needed to determine the moment at which the TTC1 $(x)$ information used to control the ball-propulsion movement is picked up. Here we formed two hypotheses: (a) the action is planned before the movement and in this case the pickup of information occurs before the initiation of the movement (Hypothesis $1 ; \mathrm{H} 1$ ) or (b) the action is still controlled during the course of execution, and information is still being used during the propulsion movement (Hypothesis 2; H2). Arbitrarily, we chose as the reference point for error prediction the initiation of the movement (the instant the ball is released from the 5-cm switch). According to $\mathrm{H} 1$, one would expect that the predicted error would be less than the observed error because there is a time lag between the pickup of $\operatorname{TTC} 1(x)$ information and the initiation of movement. This lag induces an increase in the estimated arrival time (and thus an increase of the measured errors compared with our predictions).

\footnotetext{
${ }^{7}$ For each participant, this occlusion occurred at least $100 \mathrm{~ms}$ after the ball release. In order to verify that the occlusion did not lead to degraded performances, a complementary experiment was conducted to compare the participants' precision in two presentation conditions (occluded and none occluded). The analysis revealed no effect of occlusion. The participants performed equivalently between the two presentation conditions and thus occlusion had no effect on precision (Benguigui, 1997).
} 


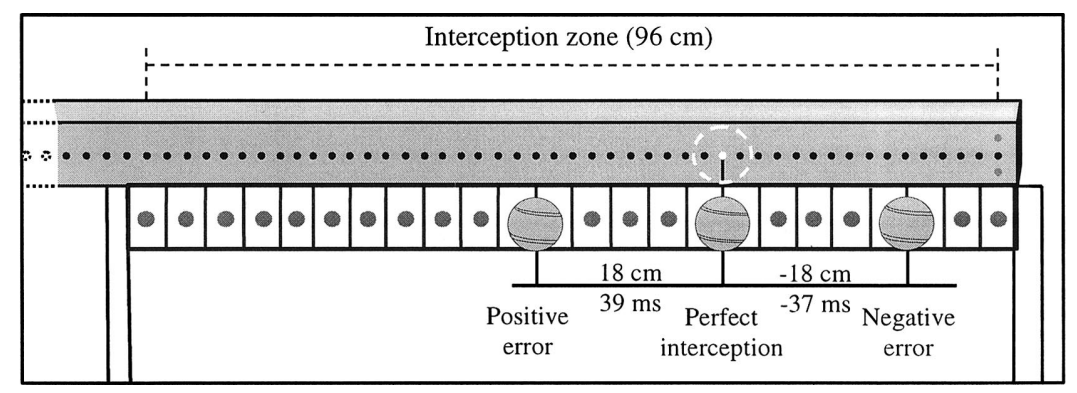

Figure 7. View of the interception zone, illustrating the calculation of errors with Trajectory Condition 9 (initial velocity of stimulus: $2.15 \mathrm{~m} / \mathrm{s}$; acceleration: $2.4 \mathrm{~m} / \mathrm{s}^{2}$ ) in Experiment 2: (a) positive error, in which the contact of the ball with the end of the table occurs at a point $18 \mathrm{~cm}$ beyond the current location of the stimulus (here the temporal error is $39 \mathrm{~ms}$ ); (b) perfect interception, in which the temporal error is 0 ; and (c) negative error, in which the contact of the ball with the end of the table occurs at a point $18 \mathrm{~cm}$ in front of the current location of the stimulus (here the temporal error is $-37 \mathrm{~ms}$ ). Note that the participants could intercept the moving stimulus anywhere within the interception zone.

On the other hand, according to $\mathrm{H} 2$, predicted errors would be greater than measured errors because at the moment for which we predicted the error, the participant has not yet completed his use of $\operatorname{TTC} 1(x)$ information. Between the time of movement initiation (our moment of error prediction) and actually releasing the ball, the difference between $\operatorname{TTC} 1(x)$ and actual $\operatorname{TTC}(x)$, that is, TTC2 $(x)$, diminishes and thus during this time the resulting measured error will diminish. This can be seen in Figures 1 and 5, in which the difference between TTC2 $(x)$ and TTC1 $(x)$ diminishes as the moment of contact draws near. In a TTC1 $(x)$ strategy, the earlier the information determining the action is defined in any trial, the greater will be the error.

The TTC2 $(x)$ and the $\operatorname{TTC} 1(x)$ of the stimulus with respect to the point of interception at the instant of initiation of the propulsion movement was calculated for each trial. The difference between $\operatorname{TTC} 2(x)$ and $\operatorname{TTC} 1(x)$ at this moment corresponds to the predicted error for a $\operatorname{TTC} 1(x)$ strategy. A regression analysis was performed for each participant by taking predicted error as the independent variable and the $\mathrm{CE}$ as the dependent variable. The results showed that the $r^{2}$ values for individual participants range from .68 to .81 (Table 4, left half; Figure 9). The same analysis for the trajectories that had a suprathreshold $v_{\text {ratio }}$ showed similar results (Table 4, right half; Figure 9, full circle). These scores are

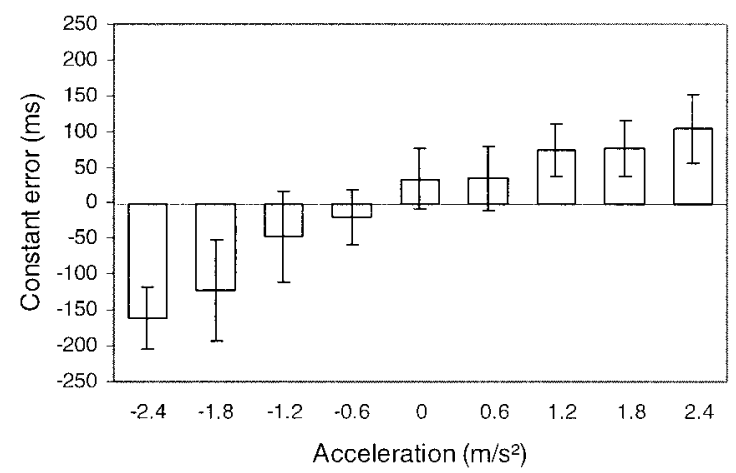

Figure 8. Constant errors as a function of the different acceleration values in Experiment 2. Bars represent the intraparticipant variability. particularly high when one takes into account that some of the variability of the responses is connected specifically with the motor component of the propulsion. Even though variability was presumably reduced somewhat during the practice trials, the propulsion movement was a source of variability both spatially (the point of contact does not always correspond perfectly with the point targeted) and temporally (the movement time does not necessarily correspond to the time planned by the actor). ${ }^{8}$ Thus the robustness of the results against the variability specific to the propulsion movement reinforces the hypothesis of the use of a $\operatorname{TTC} 1(x)$ strategy.

In addition, the slopes of the regression lines are much greater than 1.00 (from 1.49 to 2.32 ). This means that the participants committed errors greater than those predicted by the difference between $\operatorname{TTC} 1(x)$ and TTC2 $(x)$ at the initiation of the movement. It therefore appears, consistent with $\mathrm{H} 1$, that the pickup of information occurs before the initiation of the propulsion and that the movement is organized prescriptively, before the initiation of the movement.

Instant of ultimate TTC1(x) information pickup. To better understand the temporal organization of the action, we sought to identify the instant corresponding to the final pickup of information about TTC1 $(x)$ and the planning of action for each participant. It is apparent from the foregoing analysis that participants picked up the information specifying $\operatorname{TTC} 1(x)$ before initiating the pro-

\footnotetext{
${ }^{8}$ It is interesting to observe that the dispersion of the data around the linear regression was quite similar in the first and in the second experiment. A student $t$ test showed no significant difference between the $r^{2}$ transformed to a Fisher $z$ in the two tasks, $t(14)=-0.72, p>.05$, with mean $r^{2}$ values of $.68( \pm .16)$ and $.75( \pm .05)$, respectively. These results suggest therefore that the variability generated by the temporal extrapolation of the PM task was equivalent to the variability generated by the motor component of the indirect interceptive task. Regarding the third difference in these tasks (i.e., fixed or free interceptive point), it is difficult to say which condition makes the task easier. The increased task degrees of freedom does not necessarily make the task more difficult; indeed, in naturalistic settings, increased degrees of freedom may ultimately make tasks easier as they allow greater actor adaptability.
} 
pulsion movement. We tried to estimate this moment by comparing measured errors with predicted TTC1 $(x)$ errors calculated for different times before the initiation of movement. We thus calculated new predicted errors for each participant and for each trial by going backward in time from the initiation of the propulsion movement with steps of $10 \mathrm{~ms}$ to look for the moment when the predicted errors were big enough to obtain a slope of 1.00 (see Figure 10)

The predicted errors fitted the committed errors (slopes equal to 1.00) for times between 150 and $270 \mathrm{~ms}$ before the initiation of the propulsion movement (195-ms mean over all the participants; see Table 5). This means that in this type of task, the participants picked up the information necessary to plan their actions on average $195 \mathrm{~ms}$ before the beginning of the movement. One can attribute this duration to a visuomotor delay during which the response would be organized. This visuomotor delay could be defined as the latency of the central nervous system during which no information perceived in the environment can be used to produce the interceptive action (e.g., Brenner, Smeets, \& de Lussanet, 1998; Savelsbergh, Whiting, \& Bootsma, 1991; Tresilian, 1993; Whiting \& Sharp, 1974).

Our estimate of the delay is greater than that found in some studies on the regulation in interceptive actions (e.g., Bootsma \& van Wieringen, 1990; Lee et al., 1983; Savelsbergh et al., 1991; Whiting, Gill, \& Stephenson, 1970), but it conforms to others (e.g., Brenner et al., 1998; P. McLeod, 1987; Smeets, Brenner, \& de Lussanet, 1998) and particularly to that of Michaels et al. (2001) in the initiation of the punching action (between 150 and $250 \mathrm{~ms}$ ). ${ }^{9} \mathrm{It}$ is possible that the visuomotor delays are longer when the information serves to initiate the action than when it serves to control an action in progress (Michaels et al., 2001). This hypothesis comports with the results of other studies that suggest that the delay is a function of the kind of task, the intention, and preparedness of the performer (e.g., Carlton, 1992; Zelaznik, Hawkins, \& Kisselburgh, 1987). In the present task, one may suppose that the $\operatorname{TTC} 1(x)$ information serves effectively to initiate the movement or

Table 4

Results of the Different Regression Analyses Between Predicted Errors and Measured Errors for Each Participant in Experiment 2

\begin{tabular}{|c|c|c|c|c|c|c|}
\hline \multirow[b]{2}{*}{ Participant } & \multicolumn{3}{|c|}{$\begin{array}{l}\text { AllTr: constant errors- } \\
\text { predicted errors }\end{array}$} & \multicolumn{3}{|c|}{$\begin{array}{l}\text { Tr30: constant errors- } \\
\text { predicted errors }\end{array}$} \\
\hline & Slope & Intercept & $r^{2}$ & Slope & Intercept & $r^{2}$ \\
\hline 1 & 1.99 & 36 & .73 & 2.00 & 42 & .81 \\
\hline 2 & 1.66 & 33 & .72 & 1.67 & 39 & .77 \\
\hline 3 & 1.75 & 80 & .77 & 1.77 & 85 & .86 \\
\hline 4 & 1.60 & -44 & .75 & 1.64 & -37 & .82 \\
\hline 5 & 1.65 & 33 & .72 & 1.67 & 37 & .80 \\
\hline 6 & 2.39 & 29 & .81 & 2.59 & 48 & .90 \\
\hline 7 & 1.83 & 73 & .68 & 1.91 & 89 & .81 \\
\hline 8 & 1.64 & 26 & .82 & 1.70 & 41 & .89 \\
\hline$M$ & 1.81 & 33 & .75 & 1.87 & 43 & .83 \\
\hline$S D$ & 0.27 & 37 & .05 & 0.32 & 38 & .05 \\
\hline
\end{tabular}

Note. The error calculations use as the instant of reference the initiation of the propulsion movement. AllTr $=$ all trajectories; $\operatorname{Tr} 30=$ trajectories with an absolute $v_{\text {ratio }}$ value greater than $30 \%$.

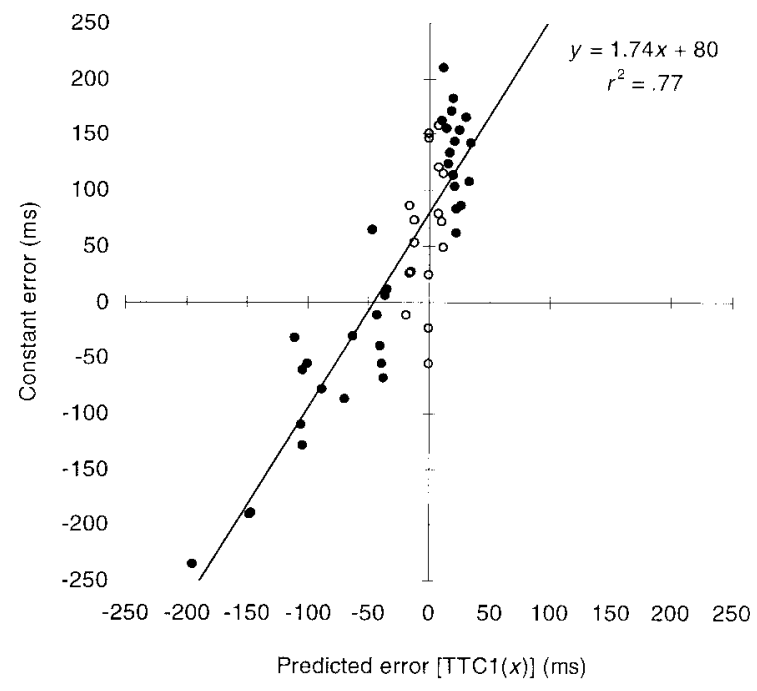

Figure 9. Constant errors as a function of predicted errors for Participant 3 (who produced typical values) in Experiment 2. The full circles correspond to trajectories that had an absolute $v_{\text {ratio }}$ greater than $30 \%$. The empty circles correspond to trajectories that had an absolute $v_{\text {ratio }}$ less than $30 \%$. The predictions of errors were calculated by taking as an instant of reference the initiation of the propulsion. TTC $=$ time to contact; $x=$ the distance between the stimulus and the target.

even to plan the movement in its entirety. If this were the case, then this implies that the visuomotor delay is integrated in the planning of the action. The actors estimate temporally the ensemble of motor sequences, including the visuomotor delay, the time of movement, and the time of the ball's trajectory, and initiate this sequence at the instant (e.g., $195 \mathrm{~ms}$ before actual ball release) when $\operatorname{TTC} 1(x)$ would become equal to the duration of this sequence.

This interpretation implies control of the movement that is exclusively predictive. Even though this hypothesis has been much disputed in recent studies of the control of interceptive actions (e.g., Bootsma et al., 1997; Dessing, Bullock, Peper, \& Beek, 2002; Lee et al., 1983; Montagne et al., 2001; Peper, Bootsma, Mestre, \& Bakker, 1994; Savelsbergh et al., 1991), it must be the case that the specific characteristics of this task require a prediction, as control of the action is not possible after releasing the ball.

\footnotetext{
${ }^{9}$ Michaels et al. (2001, Footnote 4) preferred to avoid defining this delay as a reaction time or as a perceptual-motor delay and proposed, rather, an interval. If the term reaction time is inappropriate because in an action like this the information does not appear in a discrete mode but in a continuous mode, the term perceptual-motor delay seems better suited than the term interval. Even though relatively imprecise, this term defines that to which this duration corresponds, that is, a delay necessary for the information that is picked up by the perceptual system to be used by the motor system. Michaels et al. also avoid the term perceptual-motor delay, as it suggests minimization and fixed amplitude. Even though we share these authors' idea that the delay can vary from one individual and situation to another as a function of task constraints, level of attention given to the source of information, and the intention of the actor and individual factors, we do not see anything in the term perceptual-motor delay that would prevent translating this variability.
} 


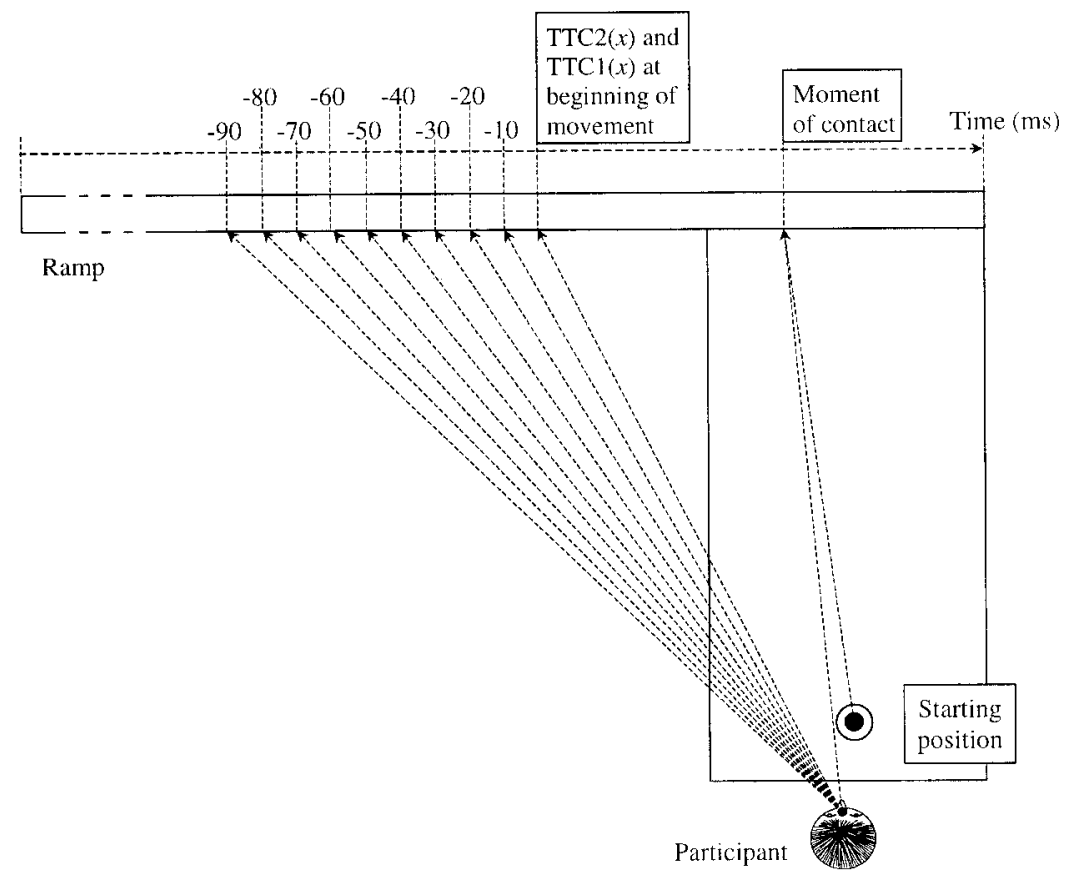

Figure 10. Illustration of the steps used to calculate $\operatorname{TTC} 2(x)$ and $\operatorname{TTC} 1(x)$ in the instants preceding the initiation of the propulsion in Experiment 2; $x$ corresponds to the distance between the stimulus and the target. The calculations of $\operatorname{TTC} 2(x)$ and $\operatorname{TTC} 1(x)$ were performed for each trial by going backward in time from the moment of movement initiation by using steps of $10 \mathrm{~ms}$ in order to obtain a calculation of the errors that participants should have produced. New predictions were made assuming that information was picked up $10 \mathrm{~ms}$, $20 \mathrm{~ms}, 30 \mathrm{~ms}$, and so on, before the beginning of the movement. TTC = time to contact.

The very short duration of the propulsion, usually less than $100 \mathrm{~ms}$ (on average $57 \mathrm{~ms}$ over all the participants) could explain the absence of control during the execution of the movement.

Using a similar indirect interceptive task, Fleury, Bard, Teasdale, Michaud, and Lamarre (1999) showed that even with longer movement $(\approx 250 \mathrm{~ms})$, the ball release occurred near peak hand

Table 5

Results of the Different Regression Analyses for Each Participant and for the Ensemble of Data Between Predicted Errors and Measured Errors

\begin{tabular}{ccccc}
\hline & \multicolumn{4}{c}{ Constant errors-predicted errors } \\
\cline { 2 - 5 } Participant & $\begin{array}{c}\text { Delay for which } \\
\text { slope }=1.00(\mathrm{~ms})\end{array}$ & Slope & Intercept & $r^{2}$ \\
\hline 1 & 220 & 1.00 & 22 & .72 \\
2 & 150 & 1.00 & 25 & .66 \\
3 & 190 & 1.00 & 58 & .79 \\
4 & 170 & 1.00 & -56 & .75 \\
5 & 150 & 1.00 & 25 & .70 \\
6 & 270 & 1.00 & 15 & .86 \\
7 & 220 & 1.00 & 58 & .71 \\
8 & 190 & 1.00 & 14 & .83 \\
$M$ & 195 & & & .75 \\
$S D$ & 41 & & 35 & .07 \\
\hline
\end{tabular}

Note. The error calculations use as the instant of reference the moment the slope of the regression lines becomes equal to 1 . velocity during the throwing movement. This result was interpreted by Fleury et al. as evidence of a ballistic-type of control. Another interesting result from this study focused on the performance of a deafferented subject who had a total loss of touch, vibration, pressure, and kinesthesia of her four limbs. Overall, the results showed that she was able, by using a predictive strategy, to have rather good timing accuracy in an indirect interceptive task without any hand sensory feedback (proprioception or vision).

In sum, it can be inferred that indirect interceptive actions involve a ballistic-type (e.g., Shea, 1980) or impulse-type of control (Tresilian, 1995). The intervention of mechanisms permitting regulation of the movement during its execution could be therefore excluded (e.g., Schmidt \& Russel, 1972).

Analysis of the movement data. Two variables were measured to account for the action produced by the participants: the movement time (MT) between the initiation of the propulsion movement and the moment of release, and the total time (TT) of the propulsion between the initiation of the movement and the ball's contact at the end of the table. For each of these variables, the mean (MTM and TTM) and standard deviation (MTSD and TTSD) were calculated for each participant and for each acceleration condition, and repeated measures ANOVAs were performed for each dependent variable, with acceleration as an independent variable.

Results for MTM showed no effect of acceleration, $F(8,56)=$ $0.41, p>.05$, nor for MTSD, $F(8,56)=1.07, p>.05$. The mean value of MTM obtained over all the participants was $57 \mathrm{~ms}( \pm 16$ $\mathrm{ms})$. The ANOVA on the TTM revealed no effect of acceleration, $F(8,56)=1.52, p>.05$, nor for TTSD, $F(8,56)=1.95, p>.05$. 
The mean value of TTM obtained across participants was $373 \mathrm{~ms}$ ( $\pm 34 \mathrm{~ms}$ ).

The lack of effect of the different trajectories used on MTM and TTM could suggest that propulsion actions are initiated on the basis of a temporal variable that attains a critical value at the moment of initiation or in the instants preceding that moment. In order to test this hypothesis, we performed an analysis of the same type as that done by Michaels et al. (2001). The principle of this analysis is to analyze the evolution of different information variables in the instants preceding the initiation of the movement in order to identify the appearance of a critical value for one of the variables which would explain the stability of the movement times. Three optic variables $(\theta, \dot{\theta}$, and $\ddot{\theta})$ and three temporal variables (TTC1 $[x]$, TTCI $[x]$, and TTC2 $[x]$ ) were chosen for this analysis (see Figure 2).

In this analysis, the evaluation of these variables for each of the nine trajectories, and for each trajectory by 10 -ms steps, was compared with the time remaining before the initiation of the propulsion movement. An example is given with the results of Participant 3 (see Figure 11). For this analysis, no point of convergence of the data appears for $\theta, \ddot{\theta}$, TTCI $(x)$, and TTC2 $(x)$. The evolution for $\operatorname{TTC} 1(x)$ as for $\dot{\theta}$, however, is marked by a point of convergence that appears in the instants preceding the initiation of the movement. In order to identify this point of convergence, we performed ANOVAs for each participant, each variable, and each step of the analysis with acceleration as an independent variable. The $p$ values were used as informal indices of the smallest difference between the trajectories. The $p$ values allow the quantitative localization of the point of convergence of the data (see Figure 11). ${ }^{10}$ The results over all the participants indicate intervals ranging from 150 to $280 \mathrm{~ms}$ for TTC1(x) and from 180 to $300 \mathrm{~ms}$ for $\dot{\theta}$ (see Table 6).

The proximity of the observations for $\operatorname{TTC} 1(x)$ and for $\dot{\theta}$ could be the result of the high correlations that exist between these two variables, which are from .87 to 1.00 for the 8 participants. This covariation poses some difficulties for deciding between the participants' use of the one or the other of these variables, all the more so given the reasonable arguments for either. Consider our $\dot{\theta}$ results and those of Michaels et al. (2001) on the use of looming $(\dot{r})$ information. On the one hand, these results suggest that the velocity of constriction of the angle $\theta$ could be used for initiating the movement. However, the use of $\operatorname{TTC} 1(x)$ information cannot be rejected, because $\dot{\theta}$ (or $\dot{r}$ ) does not give information about the time remaining before the arrival of the stimulus at the interception point. On the one hand, one possibility is that the action could be planned with a combined use of $\dot{\theta}$ (or $\dot{r}$ ), to initiate the action, and $\operatorname{TTC} 1(x)$ information, to plan the duration of the action. On the other hand, if $\operatorname{TTC} 1(x)$ information is indispensable for the timing of action, it should also be sufficient for initiating the action. It would be uneconomical for the perceptual system to use two different forms of information when one would be sufficient for action. Although some authors suggest the possibility of using different sources of information for the same action (Cutting, 1986; Michaels et al., 2001; Tresilian, 1999a), we believe that TTC1 $(x)$ information is the variable used for initiating the propulsion and timing of action.

In addition, it must be noted that there is a close proximity between visuomotor delays obtained in analysis of the errors (see Table 5) and in analysis performed on the initiation of movement with $\operatorname{TTC} 1(x)$ (see Table 6). A regression analysis between the visuomotor delays obtained from the two different analyses for each of the participants shows an $r^{2}$ of .90 and a slope of 1.06 .

Another point in the framework of this new analysis concerns the planning of the action on the basis of $\operatorname{TTC} 1(x)$ information. The variability observed with respect to the level of the critical value of $\operatorname{TTC} 1(x)$ (which ranged from 37 to $64 \mathrm{~ms}$; see Table 6) could suggest that this value does not condition completely the propulsion but that the participants adjust their movement with respect to this value or to some other information. However, a student $t$ test reveals that this variability is greater than that at the level of TT, ranging from 18 to $48 \mathrm{~ms}$ (see Table 6 ), $t(7)=12.93$, $p<.05$. This observation suggests that there is no specific adjustment of the movement with respect to the value of $\operatorname{TTC} 1(x)$ and that the movement is produced at a constant time. Otherwise, the variability for TT should have been equal to or greater than the variability for $\operatorname{TTC} 1(x)$. This idea is also confirmed by the analyses that have been made between the value of TTC1 $(x)$ and TT for each participant and each trial that demonstrate no significant correlation. ${ }^{11}$ These results suggest therefore that the variability at the level of the critical value of $\operatorname{TTC} 1(x)$ is connected strictly to perceptual mechanisms, just as the variability at the level of TT is connected to motor mechanisms.

\section{General Discussion}

In the course of these two experiments, it was possible to show that the participants produced their temporal estimates-in a PM task - and produced their actions - in an indirect interceptive task - on the basis of $\operatorname{TTC} 1(x)$ information. If these results are in agreement with those reported within the perspective of Lee's (1976, 1980) work (Bootsma \& Oudejans, 1993; Dubrowski \& Carnahan, 2000, 2002; Kaiser \& Hecht, 1995; Lee et al., 1983, 1997; Port et al., 1997; Ripoll \& Latiri, 1997), then they underscore the robustness of the quantitative predictions for the use of a $\operatorname{TTC} 1(x)$ strategy.

Concerning the identification of the potential information that is used for extrapolating occluded trajectories and intercepting moving objects at a certain distance, the optic quantity $\tau(\varphi, \theta)$ defined by Bootsma et al. (1997) seems to be a good candidate. Indeed, this optic quantity specifies $\operatorname{TTC} 1(x)$ in situations of tangential approach. Nevertheless, as this information was not really manipulated in these experiments, it is not possible to provide a definitive demonstration of its use.

These results clearly demonstrate that humans are not capable, in the tasks we used, of using information about the acceleration. This incapacity for using such information is consistent with observations of Todd (1981), according to which humans would

\footnotetext{
${ }^{10}$ Using an ANOVA in which the $p$ values are used for differentiating is rare and counter to the statistical principles of ANOVA, which consist uniquely of validating or rejecting a null hypothesis. This analysis is nevertheless presented here because it completes the descriptive analysis aimed at identifying the points of convergence (see Michaels et al., 2001, for a similar analysis).

${ }^{11}$ Similar analyses were made with the variables $\theta, \dot{\theta}, \dot{r}, \operatorname{TTC} 1(x)$, TTC2 $(x)$, and TTC1 $(x)$. None of these variables showed a significant correlation.
} 


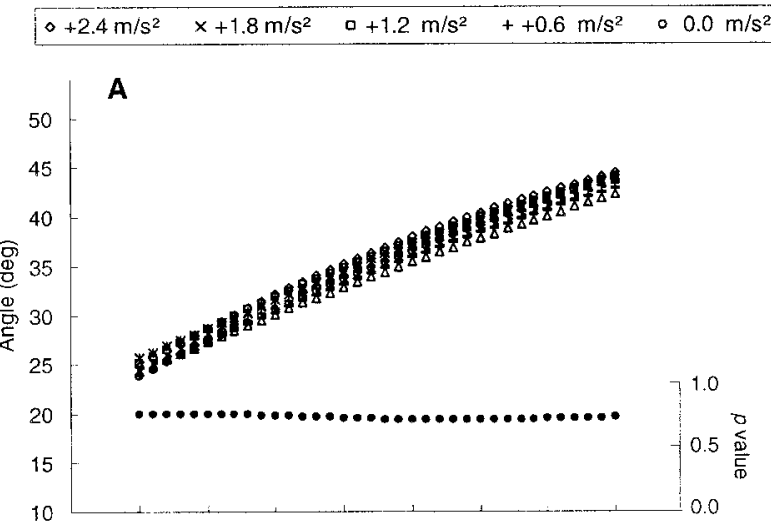

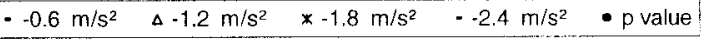
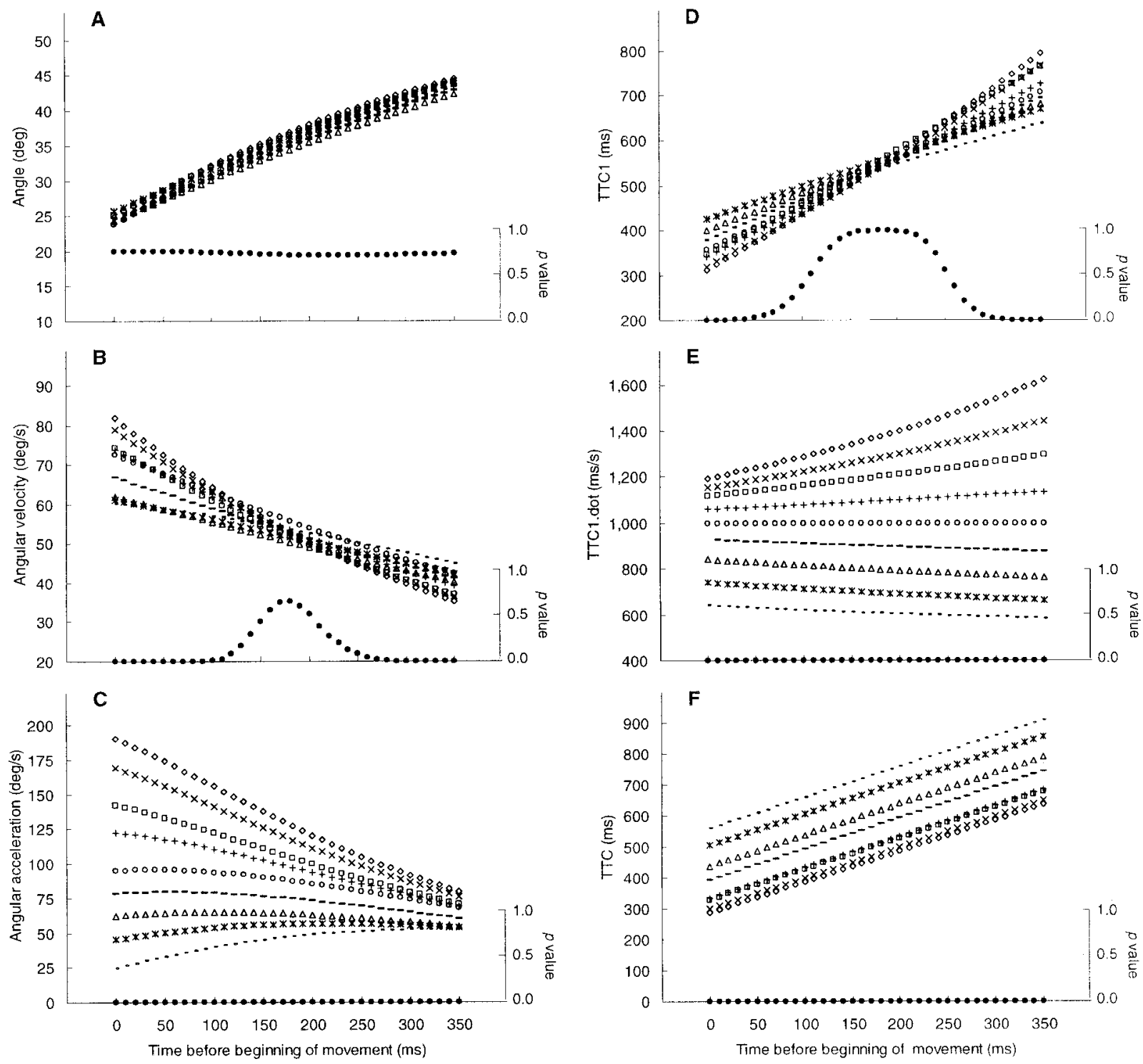

Figure 11. The evolution of the values of the angle $\theta(\mathrm{A})$, the angular velocity $\dot{\theta}(\mathrm{B})$, the angular acceleration $\ddot{\theta}(\mathrm{C}), \operatorname{TTC} 1(x)(\mathrm{D}), \operatorname{TTC} 1(x)(\mathrm{E})$, and TTC $(x)$ (which is equal to TTC2[x]; F) for Participant 3 (who had typical values) in Experiment 2; $x$ corresponds to the distance between the stimulus and the target. Each variable is presented as a function of the time remaining before the initiation of the movement $(t=0)$. There appears to be a point of convergence for $\ddot{\theta}$ and $\operatorname{TTC} 1(x)$. The $p$ values of the analysis of variance were used to identify the point at which the convergence was higher. $\operatorname{deg}=$ degree; TTC $=$ time to contact.

not have access to all of the information potentially available (e.g., the information defined by Bootsma \& Peper, 1992, or by Tresilian, 1994a).

It appears that the incapacity to use information about acceleration is independent of the $v_{\text {ratio }}$ identified in studies of perceptual discrimination and confirms the hypothesis that acceleration detection is not implicated in tasks requiring the determination of a variable specifying TTC (Dubrowski \& Carnahan, 2000, 2002). These results equally confirm the observations of Werkhoven et al. (1992) that the visual system is not equipped with sensors allowing direct detection of acceleration but operates, in discrimination tasks, by successive comparisons in time of the stimulus's velocity.

The results also show that possible detection of acceleration does not allow a minimization of errors and that the findings of McIntyre et al. (2001) are specific to situations of free fall in which acceleration is due to gravity or in a situation in which actors are always confronted with a very limited set of trajectories.

It is left for us to wonder about the repercussions of having this inability to use information about acceleration in interceptive 
Table 6

Mean Values (and Standard Deviations) of TTC1(x) and of the Angular Velocity $(\dot{\theta})$ for Which a Point of Convergence Is Observed With the Corresponding Visuomotor Delay

\begin{tabular}{cccccc}
\hline & $\begin{array}{c}\text { TTC1 }(x) \text { at } \\
\text { beginning of } \\
\text { movement }\end{array}$ & $\begin{array}{c}\text { Visuomotor } \\
\text { delay }\end{array}$ & $\begin{array}{c}\text { Angle } \\
\text { velocity }\end{array}$ & $\begin{array}{c}\text { Visuomotor } \\
\text { delay }\end{array}$ & TT \\
\hline 1 & $569(62)$ & 240 & $48.9(4.5)$ & 260 & $360(48)$ \\
2 & $485(61)$ & 150 & $51.1(7.3)$ & 210 & $370(41)$ \\
3 & $534(49)$ & 180 & $52.8(4.2)$ & 180 & $432(31)$ \\
4 & $623(55)$ & 200 & $44.7(4.1)$ & 210 & $377(29)$ \\
5 & $507(47)$ & 150 & $47.9(3.8)$ & 190 & $392(32)$ \\
6 & $539(37)$ & 280 & $50.5(3.0)$ & 300 & $284(18)$ \\
7 & $601(64)$ & 230 & $48.3(5.6)$ & 230 & $439(40)$ \\
8 & $607(59)$ & 220 & $47.1(4.2)$ & 230 & $410(35)$ \\
$M$ & 558 & 206 & 48.9 & 226 & 373 \\
$S D$ & 50 & 45 & 2.5 & 39 & 34 \\
\hline
\end{tabular}

Note. Angle velocity values represent degrees per second; all other values represent milliseconds. The standard deviations for all variables were calculated for each participant on the totality of the 54 trials. TTC $=$ time to contact; TT $=$ total time of propulsion.

timing. TTC1 $(x)$ information becomes approximate when the actor does not have the possibility to pick up and use continuously information up to the object's arrival. In the two tasks used, the errors were up to $800 \mathrm{~ms}$ for the higher accelerations. This corresponds to a basic inability of the perception-action system to detect and use information about acceleration. In the kinds of indirect interceptive actions one finds in sports, acceleration of the moving object creates difficulties for precise production of effective action. To play basketball or football, for example, which requires long passes to moving teammates, or in trap shooting, the acceleration of the target (teammate or clay pigeon, respectively) would certainly be a source of error. Nevertheless, it is clear that expert basketball and football players and marksmen are capable of taking account of these variations, not necessarily in terms of psychophysical detection, but by learning some method of aiming and acting which allows them to minimize errors susceptible to be produced.

Our experiments were designed to eliminate the possibility of the participants using such compensatory strategies. Even if some of the values of acceleration used in these two experiments were detectable (sufficient $v_{\text {ratio }}$ ), the trajectories were not experienced constantly but presented in a random order and no KR was provided. Both conditions were selected to test the basic ability to use information about acceleration but prevented any adaptations from participants. It would be particularly interesting to study the adaptations possible in such a task selected in order to compensate for the incapacity of the perceptual system to take account directly of acceleration. It would be possible to test whether people are able to develop, in specific situations, some specific adaptations such as the use of an internal model of the acceleration, the use of other cues (e.g., angular or distance information), or a diminution of the throwing movement time, which would allow more time to use target trajectory information. Our results and the specificity of the task open a field of research that could enable us to understand better the learning process when the basic functions of the visual system are not sufficient to solve a problem.

It was also possible to show in the indirect interceptive task that the participants organized their responses in an exclusively pre- scriptive mode (Dubrowski, Lam, \& Carnahan, 2000; Fleury et al., 1999). Moreover, the results indicated that the propulsive actions were planned at a constant time around $200 \mathrm{~ms}$ before the initiation of the action. Because they were at critical values, two variables$\operatorname{TTC} 1(x)$ information and $\dot{\theta}$-were identified as candidates for information used in the initiation of action. The correlation of these two variables did not allow definitive separation of them in favor of one or the other. The use of $\dot{\theta}$ to initiate the action to complement the use of information specifying TTC1 $(x)$ to plan the action would be consistent with the recent results of Michaels et al. (2001), which suggest that interceptive actions could be controlled on the basis of different sources of information. Nevertheless, if information specifying $\operatorname{TTC} 1(x)$ is used for planning the duration of the action, it would be surprising if it is not used also for initiating the action. It appears therefore reasonable to conclude that $\operatorname{TTC} 1(x)$ information is also used for initiation of the propulsion movement.

Our finding of the initiation of the action at a constant time value is in contradiction with a good deal of empirical research showing that the timing of movement initiation is not a critical aspect of the task (e.g., Lee et al., 1983; Tresilian \& Lonergan, 2002). However, such previous observations have been made in tasks in which online regulations were possible. In indirect interceptive tasks, online regulations are not possible. The timing of initiation becomes therefore a critical aspect of the task. A good way for the participant to simplify the problem would be to initiate action for a constant value of $\operatorname{TTC} 1(x)$. Thus it is apparent how task constraints can determine the used strategy.

Although they may be noteworthy, the results of the prescriptive control of indirect interceptive action do not call into question the importance of online regulation mechanisms, because without them the production of a precise action would be impossible whenever the velocity of the object is not constant (Bootsma et al., 1997; Dessing et al., 2002; Montagne et al., 2000; Peper et al., 1994). Placed in a more general context, these results suggest that the control of interceptive actions occurs according to a sequential mode, with, first, a prescriptive organization of the action allowing a priori determination of the interception zone and the global 
spatiotemporal organization of the response to be produced, and second, regulation during the course of execution allowing adjustment of the action as a function of TTC1. The relative importance of these two control modes would depend directly on the constraints of the task and, more precisely, on the possibility or impossibility of regulating effector action in the instants preceding contact with the object, as well as the precision required for the action.

\section{References}

Babler, T. G., \& Dannemiller, J. L. (1993). Role of image acceleration in judging landing location of free-falling projectiles. Journal of Experimental Psychology: Human Perception and Performance, 19, 15-31.

Benguigui, N. (1997). Effet de la pratique d'un sport de balle sur le développement des processus perceptifs impliqués dans les actions d'interception [Effects of tennis practice on the coincidence timing accuracy of adults and children]. Unpublished doctoral dissertation, University of Poitiers, Poitiers, France.

Benguigui, N., Ripoll, H., \& Broderick, M. P. (2002). Intercepting accelerating objects. In K. Davids, G. Savelsbergh, S. Bennett, \& J. Van Der Kamp (Eds.), Dynamic interception actions in sport: Current research and practical application (pp. 158-171). London: Routledge.

Bootsma, R. J., Fayt, V., Zaal, F. T. J. M., \& Laurent, M. (1997). On the information-based regulation of movement. Journal of Experimental Psychology: Human Perception and Performance, 23, 1282-1289.

Bootsma, R. J., Marteniuk, R. G., \& MacKenzie, C. L. (1991). Perception of time-to-contact for actions of different duration. In P. J. Beek, R. J. Bootsma, \& P. C. W. van Wieringen (Eds.), Studies in perception and action: Proceedings from the 6th International Conference on Event Perception and Action (pp. 188-192). Amsterdam: Rodopi.

Bootsma, R. J., \& Oudejans, R. R. D. (1993). Visual information about the time to collision between two objects. Journal of Experimental Psychology: Human Perception and Performance, 19, 1041-1052.

Bootsma, R. J., \& Peper, C. E. (1992). Predictive visual information sources for the regulation of action with special emphasis on catching and hitting. In L. Proteau \& D. Elliott (Eds.), Vision and motor control (pp. 285-314). Amsterdam: Elsevier Science.

Bootsma, R. J., \& van Wieringen, P. C. W. (1990). Timing an attacking forehand drive in table tennis. Journal of Experimental Psychology: Human Perception and Performance, 16, 21-29.

Brenner, E., Smeets, J. B., \& de Lussanet, M. H. (1998). Hitting moving targets: Continuous control of the acceleration of the hand on the basis of the target's velocity. Experimental Brain Research, 122, 467-474.

Brouwer, A.-M., Brenner, E., \& Smeets, J. B. J. (2002). Perception of acceleration with short presentation time: Can acceleration be used in interception? Perception \& Psychophysics, 64, 1160-1168.

Caird, J. K., \& Hancock, P. A. (1994). The perception of arrival time for different oncoming vehicles at an intersection. Ecological Psychology, 6, $83-109$

Calderone, J. B., \& Kaiser, M. K. (1989). Visual acceleration detection: Effect of sign and motion orientation. Perception \& Psychophysics, 45, 391-394.

Carel, W. L. (1961). Visual factors in the contact analog (Report No. R61ELC60, NTIS No. 263-820). Ithaca, NY: General Electric Company Advanced Electronics Center.

Carlton, L. G. (1992). Visual processing time and the control of movement. In L. Proteau \& D. Elliott (Eds.), Vision and motor control (pp. 3-31). Amsterdam: Elsevier Science.

Cavallo, V., \& Laurent, M. (1988). Visual information and skill level in time-to-collision estimation. Perception, 17, 623-632.

Chapman, S. (1968). Catching a baseball. American Journal of Physics, 36, $868-870$
Cutting, J. E. (1986). Perception with an eye for motion. Cambridge, MA: MIT Press.

DeLucia, P. R., \& Lidell, G. W. (1998). Cognitive motion extrapolation and cognitive clocking process in prediction motion tasks. Journal of Experimental Psychology: Human Perception and Performance, 24, 901-914.

Dessing, J. C., Bullock, D., Peper, C. E., \& Beek, P. J. (2002). Prospective control of manual interceptive actions: Comparative simulations of extant and new model construct. Neural Networks, 15, 163-179.

Dubrowski, A., \& Carnahan, H. (2000). Task dependent processing of visual information about target acceleration. Brain and Cognition, 43, 172-177.

Dubrowski, A., \& Carnahan, H. (2002). Action-perception dissociation in response to target acceleration. Vision Research, 42, 1465-1473.

Dubrowski, A., Lam, J., \& Carnahan, H. (2000). Target velocity effects on manual interception kinematics. Acta Psychologica, 104, 103-118.

Fleury, M., Bard, C., Teasdale, N., Michaud, D., \& Lamarre, Y. (1999). How efficient are the central mechanisms for the learning and retention of coincident timing actions? Neuropsychologia, 37, 723-730.

Goodale, M. A., \& Milner, A. D. (1992). Separate visual pathways for perception and action. Trends in Neurosciences, 15, 20-25.

Kaiser, M. K., \& Hecht, H. (1995). Time-to-passage judgments in nonconstant optical flow fields. Perception \& Psychophysics, 57, 817-825.

Kaiser, M. K., \& Mowafy, L. (1993). Optical specification of time-topassage: Observers' sensitivity to global tau. Journal of Experimental Psychology: Human Perception and Performance, 19, 1028-1040.

Keil, D., Holmes, P., Bennett, S., Davids, K., \& Smith, N. (2000). Theory and practice in sport psychology and motor behaviour needs to be constrained by integrative modeling of brain and behaviour. Journal of Sport Sciences, 18, 433-443.

Lacquaniti, F., Carozzo, M., \& Borghese, N. (1993). The role of vision in tuning anticipatory motor responses of the limbs. In A. Berthoz (Ed.), Multisensory control of movement (pp. 379-393). Oxford, England: Oxford University Press.

Lacquaniti, F., \& Maioli, C. (1989a). Adaptation to suppression of visual information during catching. Journal of Neurosciences, 9, 149-159.

Lacquaniti, F., \& Maioli, C. (1989b). The role of preparation in tuning anticipatory and reflex responses during catching. Journal of Neurosciences, 9, 134-148.

Lee, D. N. (1976). A theory of visual control of braking based on information about time-to-collision. Perception, 5, 437-459.

Lee D. N. (1980). Visuo-motor co-ordination in space-time. In G. E. Stelmach \& J. Requin (Eds.), Tutorials in motor behavior (pp. 281-293). Amsterdam: North-Holland.

Lee, D. N., Port, N. L., \& Georgopoulos A. P. (1997). Manual interception of moving targets: II. On-line control of overlapping submovements. Experimental Brain Research, 116, 421-433.

Lee, D. N., \& Young, D. S. (1985). Visual timing in interceptive actions. In D. J. Ingle, M. Jeannerod, \& D. N. Lee (Eds.), Brain mechanisms and spatial vision (pp. 1-30). Dortrecht, the Netherlands: Martinus Nijhoff.

Lee, D. N., Young, D. S., Reddish, P. E., Lough, S., \& Clayton, T. M. H. (1983). Visual timing in hitting an accelerating ball. Quarterly Journal of Experimental Psychology: Human Experimental Psychology, 35(A), 333-346.

McIntyre, J., Zago, M., Berthoz, A., \& Lacquaniti, F. (2001). Does the brain model Newton's laws? Nature Neurosciences, 4, 693-694.

McLeod, P. (1987). Visual reaction time and high-speed ball games. Perception, 16, 49-59.

McLeod, P., Reed, N., \& Dienes, Z. (2001). Toward a unified fielder theory: What we do not yet know about how people run to catch a ball. Journal of Experimental Psychology: Human Perception and Performance, 27, 1347-1355.

McLeod, R. W., \& Ross, H. E. (1983). Optic flow and cognitive factors in time-to-collision estimates. Perception, 12, 417-423. 
Michaels, C. F., \& Oudejans, R. R. D. (1992). The optics and actions of catching fly balls: Zeroing out optical acceleration. Ecological Psychology, 4, 199-222.

Michaels, C. F., Zeinstra, E. B., \& Oudejans, R. R. D. (2001). Information and action in punching a falling ball. Quarterly Journal of Experimental Psychology: Human Experimental Psychology, 54(A), 69-93.

Montagne, G., Fraisse, F., Ripoll H., \& Laurent, M. (2000). Perceptionaction coupling in an interceptive task: First-order time-to-contact as an input variable. Human Movement Science, 19, 59-72.

Oudejans, R. R. D., Michaels, C. F., Bakker, F. C., \& Davids, K. (1999). Shedding some light on catching in the dark: Perceptual mechanisms for catching fly balls. Journal of Experimental Psychology: Human Perception and Performance, 25, 531-542.

Peper, C. E., Bootsma, R. J., Mestre, D. R., \& Bakker, F. C. (1994). Catching balls: How to get the hand to the right place at the right time. Journal of Experimental Psychology: Human Perception and Performance, 20, 591-612.

Port, N. L., Lee, D., Dassonville, P., \& Georgopoulos, A. P. (1997). Manual interception of moving targets: I. Performance and movement initiation. Experimental Brain Research, 116, 406-420.

Regan, D. (1997). Visual factors in hitting and catching. Journal of Sport Sciences, 15, 533-558.

Regan, D. M., Kaufman, L., \& Lincoln, J. (1986). Motion in depth and visual acceleration. In K. R. Boff, L. Kaufman, \& J. P. Thomas (Eds.), Handbook of human perception and human performance: Vol. 1. Sensory processes and perception (pp. 19-1-19-46). New York: Wiley.

Ripoll, H., \& Latiri, I. (1997). Effect of expertise in a fast ball game on coincident-timing accuracy. Journal of Sport Sciences, 15, 573-580.

Rosenbaum, D. A. (1975). Perception and extrapolation of velocity and acceleration. Journal of Experimental Psychology: Human Perception and Performance, 1, 395-403.

Savelsbergh, G. J. P., Whiting, H. T. A., \& Bootsma, R. J. (1991). "Grasping" tau. Journal of Experimental Psychology: Human Perception and Performance, 17, 315-322.

Schiff, W., \& Detwiler, M. (1979). Information used in judging impending collision. Perception, 8, 647-658.

Schiff, W., \& Oldak, R. (1990). Accuracy of judging time to arrival: Effects of modularity, trajectory, and gender. Journal of Experimental Psychology: Human Perception and Performance, 16, 303-316.

Schmidt, R. A., \& Russel, D. G. (1972). Movement velocity and movement time as determiners of degree of preprogramming in simple movement. Journal of Experimental Psychology, 96, 315-320.

Shea, C. H. (1980). Effects of extended practice and movement time on the control of a coincident timing task. Research Quarterly for Exercise and Sport, 51, 369-381.

Sidaway, B., Fairweather, M., Sekiya, H., \& McNitt-Gray, J. (1996). Time-to-collision estimation in a simulated driving task. Human Factors, 38, 101-113.

Smeets, B. J., Brenner, E., \& de Lussanet, M. H. E. (1998). Visuomotor delays when hitting running spiders. In B. Bril, A. Ledebt, G. Dietrich,
\& A. Roby-Brami (Eds.), Advances in perception-action-coupling (pp. 36-40). Paris: EDK

Smeets, J. B. J., Brenner, E., Trébuchet, S., \& Mestre, D. R. (1996). Is judging time-to-contact based on "tau"? Perception, 25, 583-590.

Todd, J. T. (1981). Visual information about moving objects. Journal of Experimental Psychology: Human Perception and Performance, 7, 795810.

Tresilian, J. R. (1990). Perceptual information for the timing of interceptive action. Perception, 19, 223-239.

Tresilian, J. R. (1993). Four questions of time to contact: A critical examination of research on interceptive timing. Perception, 22, 653680.

Tresilian, J. R. (1994a). Approximate information sources and perceptual variables in interceptive timing. Journal of Experimental Psychology: Human Perception and Performance, 20, 154-173.

Tresilian, J. R. (1994b). Perceptual and motor processes in interceptive timing. Human Movement Science, 13, 335-373.

Tresilian, J. R. (1995). Perceptual and cognitive processes in time-tocontact estimation: Analysis of prediction motion and relative judgment tasks. Perception \& Psychophysics, 57, 231-245.

Tresilian, J. R. (1997). A revised tau hypothesis: Consideration of Wann's (1996) analysis. Journal of Experimental Psychology: Human Perception and Performance, 23, 1272-1281.

Tresilian, J. R. (1999a). Analysis of recent empirical challenges to an account of interceptive timing. Perception \& Psychophysics, 61, 3, $515-528$.

Tresilian, J. R. (1999b). Visually timed action: Time-out for "tau"? Trends in Cognitive Sciences, 3, 301-310.

Tresilian, J. R., \& Lonergan, A. (2002). Intercepting a moving target: Effects of temporal precision constraints and movement amplitude. Experimental Brain Research, 142, 193-207.

Wann, J. P. (1996). Anticipating arrival: Is the tau margin a specious theory? Journal of Experimental Psychology: Human Perception and Performance, 22, 1031-1048.

Werkhoven, P., Snippe, H., \& Toet, A. (1992). Visual processing of optic acceleration. Vision Research, 32, 2313-2329.

Whiting, H. T. A., Gill, E. B., \& Stephenson, J. M. (1970). Critical time intervals for taking in flight information in a ball-catching task. Ergonomics, 13, 265-272.

Whiting, H. T. A., \& Sharp, R. H. (1974). Visual occlusion factors in a discrete ball-catching task. Journal of Motor Behavior, 6, 11-16.

Yakimoff, N., Mateeff, S., Ehrenstein, W. H., \& Hohnsbein, J. (1993). Motion extrapolation performance: A linear model approach. Human Factors, 35, 501-510.

Zelaznik, H. N., Hawkins B., \& Kisselburgh L. (1987). The effects of movement distance and movement time on visual feedback processing in aimed hand movements. Acta Psychologica, 65, 181-191.

Received July 25, 2002

Revision received January 8, 2003

Accepted March 21, 2003 